\title{
The Inhibitory Role of Rab11b in Osteoclastogenesis through Triggering Lysosome-Induced Degradation of c-Fms and RANK Surface Receptors
}

\author{
Manh Tien Tran ${ }^{1}{ }^{1}$, Yuka Okusha ${ }^{1,2}$, Yunxia Feng ${ }^{1,3}$, Masatoshi Morimatsu ${ }^{4}$, Penggong Wei ${ }^{1,5}$,

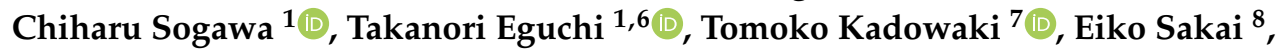 \\ Hirohiko Okamura ${ }^{9}$ D, Keiji Naruse ${ }^{4}$, Takayuki Tsukuba ${ }^{8} \mathbb{D}$ and Kuniaki Okamoto ${ }^{1, *(D)}$ \\ 1 Department of Dental Pharmacology, Graduate School of Medicine, Dentistry and Pharmaceutical Sciences, \\ Okayama University, 2-5-1 Shikata-cho, Kita-ku, Okayama 700-8525, Japan; \\ trantienmanh1508@gmail.com (M.T.T.); yokusha@bidmc.harvard.edu (Y.O.); yunxiafeng0302@163.com (Y.F.); \\ 13898129859@163.com (P.W.); caoki@md.okayama-u.ac.jp (C.S.); eguchi.takanori@gmail.com (T.E.) \\ 2 Division of Molecular and Cellular Biology, Department of Radiation Oncology, Beth Israel Deaconess \\ Medical Center, Harvard Medical School, Boston, MA 02115, USA \\ 3 College of Basic Medicine, China Medical University, Shenyang 110122, China \\ 4 Department of Cardiovascular Physiology, Graduate School of Medicine, Dentistry and Pharmaceutical \\ Sciences, Okayama University, 2-5-1 Shikata-cho, Kita-ku, Okayama 700-8558, Japan; \\ mmorimatsu@okayama-u.ac.jp (M.M.); knaruse@md.okayama-u.ac.jp (K.N.) \\ 5 Department of Endodontics, School of Stomatology, China Medical University, Shenyang 110002, China \\ 6 Advanced Research Center for Oral and Craniofacial Sciences, Graduate School of Medicine, Dentistry and \\ Pharmaceutical Sciences, Okayama University, 2-5-1 Shikata-cho, Kita-ku, Okayama 700-8525, Japan \\ 7 Department of Frontier Oral Science, Graduate School of Biomedical Sciences, Nagasaki University, \\ 1-7-1 Sakamoto, Nagasaki 852-8588, Japan; tomokok@nagasaki-u.ac.jp \\ 8 Department of Dental Pharmacology, Graduate School of Biomedical Sciences, Nagasaki University, \\ 1-7-1 Sakamoto, Nagasaki 852-8588, Japan; eiko-s@nagasaki-u.ac.jp (E.S.); tsuta@nagasaki-u.ac.jp (T.T.) \\ 9 Department of Oral Morphology, Graduate School of Medicine, Dentistry and Pharmaceutical Sciences, \\ Okayama University, 2-5-1 Shikata-cho, Kita-ku, Okayama 700-8525, Japan; hiro-okamura@okayama-u.ac.jp \\ * Correspondence: k-oka@okayama-u.ac.jp; Tel.: +81-86-235-6660
}

Received: 23 November 2020; Accepted: 6 December 2020; Published: 8 December 2020

check for updates

\begin{abstract}
Rab11b, abundantly enriched in endocytic recycling compartments, is required for the establishment of the machinery of vesicle trafficking. Yet, no report has so far characterized the biological function of Rab11b in osteoclastogenesis. Using in vitro model of osteoclasts differentiated from murine macrophages like RAW-D cells or bone marrow-derived macrophages, we elucidated that Rab11b served as an inhibitory regulator of osteoclast differentiation sequentially via (i) abolishing surface abundance of RANK and c-Fms receptors; and (ii) attenuating nuclear factor of activated T-cells c1 (NFATc-1) upstream signaling cascades, following RANKL stimulation. Rab11b was localized in early and late endosomes, Golgi complex, and endoplasmic reticulum; moreover, its overexpression enlarged early and late endosomes. Upon inhibition of lysosomal function by a specific blocker, chloroquine (CLQ), we comprehensively clarified a novel function of lysosomes on mediating proteolytic degradation of c-Fms and RANK surface receptors, drastically ameliorated by Rab11b overexpression in RAW-D cell-derived osteoclasts. These findings highlight the key role of Rab11b as an inhibitor of osteoclastogenesis by directing the transport of c-Fms and RANK surface receptors to lysosomes for degradation via the axis of early endosomes-late endosomes-lysosomes, thereby contributing towards the systemic equilibrium of the bone resorption phase.
\end{abstract}

Keywords: Rab11b; c-Fms; RANK; NFATc-1; osteoclasts; vesicular transport 


\section{Introduction}

Osteoclasts, multinucleated cells differentiated from macrophage-monocyte cells, play a critical role in bone tissue destruction [1,2]. Osteoclast differentiation, also called osteoclastogenesis, is directly induced via binding of the receptor activator of nuclear factor kappa-B ligand (RANKL) to the cell surface RANK receptor. Mechanistically, this binding stimulates six various signaling cascades essential for osteoclast differentiation including (i) the nuclear factor of activated T cells cytoplasmic-1 (NFATc-1); (ii) nuclear factor kappa B (NF-kB); (iii) phosphatidylinositol 3-kinase (PI3K/Akt); (iv) Jun N-terminal kinase (JNK); (v) extracellular signal-regulated kinase (Erk); and (vi) p38 mitogen-activated protein kinase (MAPK) [3], thereby boosting the secretion of bone-resorbing enzymes such as tartrate-resistant acid phosphatase (TRAP), Cathepsin K (CTSK), and matrix metalloproteinase 9 (MMP9), into the extracellular environment $[4,5]$. In addition, binding of monocyte/macrophage colony stimulating factor (M-CSF) to the cell surface c-Fms receptor is also essential for survival and differentiation of premature and mature osteoclasts [3,6]. The network of intracellular vesicle trafficking amongst subcellular compartments is evolutionarily conserved in eukaryotic cells [3,7].

Rab GTPases, specifically localized in integral membranes of intercellular organelles, are emerged as the central regulators of interrelated processes of membrane trafficking including budding, motility, docking, and fusion of vesicle, and are cardinal in transporting cargos to accurate destinations, cooperatively coupled to receptor signaling pathways $[7,8]$. Dynamic modification of Rab GTPases between inactive [Guanine Diphosphate (GDP)-bound] and active [Guanine Triphosphate (GTP)-bound] forms are catalyzed by specific enzymes. More particularly, guanine exchange factors (GEFs) catalyze the conversion of GDP-bound to GTP-bound form to switch on various cellular signals whereas GTPase activating proteins (GAPs) inactivate Rab GTPases via catalyzing GTP hydrolysis of GTP-bound form [9-12]. The Rab11 subfamily is structurally categorized into three closely related isoforms, Rab11a, Rab11b, and Rab25. Of these, Rab11a is copiously expressed; Rab11b is exclusively enriched in heart, brain, and testes [13], and in polarized MDCK, gastric parietal cells [14] whereas Rab25 is only found in the epithelial cells [15]. In rat osteoclasts, subcellular localization of Rab11b was identified to localize in perinuclear recycling compartments wherein functionally it was engaged in ruffled border membrane turnover and in osteoclast motility [14].

Our previous study clarified a vital role of lysosomal Rab44 as a decelerator of osteoclastogenesis via declining endogenous levels of c-Fms and RANK, followed by inactivation of NFATc-1 upstream signaling cascades through a poorly understood mechanism [16]. Moreover, our subsequent report disclosed the regulatory function of Rab27A on (i) directing transport routes of lysosome-related organelles to the ruffled border membrane, and (ii) weakening abundance of surface RANK and c-Fms receptors, thereby causing debilitation of osteoclast differentiation [17]. Recently we revealed a novel role of Rab11a in negatively regulating osteoclast differentiation mainly through down-regulating the surface abundance of c-fms and RANK receptors [18]. In this report, during the investigation of physiological roles of Rab11b for regulation of osteoclastogenesis, we also attempted to thoroughly elucidate a crucial role of lysosomes in the proteolytic degradation of c-Fms and RANK surface receptors in osteoclasts. Together, our findings stress the critical role of Rab11b for inhibition of osteoclastogenesis by directing the transport of c-Fms and RANK surface receptors to lysosomes for degradation via the axis of early endosomes-late endosomes-lysosomes; more importantly to provide a universal molecular mechanism by which Rab11b modulates osteoclastic bone resorption involved in the systemic homeostasis of boss mass and remodeling.

\section{Results}

\subsection{Rab11b Is Up-Regulated at a Late Stage of Osteoclast Differentiation}

To investigate the regulatory function of Rab11b during osteoclast differentiation, we initially screened the dynamic modifications of the mRNA and protein levels of Rab11b as well as several osteoclast markers such as c-Fos, NFATc-1, and CTSK in RAW-D cells and bone marrow-derived 
macrophages (BMMs) in the time courses of RANKL stimulation. Albeit the mRNA level of Rab11b was insignificantly altered (Figure 1A), the protein level thereof was strongly increased from day two to five (Figure 1C and Figure S1A) in RAW-D cells upon five days of RANKL stimulation. Besides, levels of c-Fos and NFATc-1 were significantly strengthened from zero to two days, but drastically decreased from day three to five (Figure 1C and Figure S1A). Similar effects on mRNA of Rab11b (Figure 1B), and protein levels of Rab11b, c-Fos, NFATc-1, and CTSK (Figure 1D and Figure S1B) were observed in BMMs upon 4 days of RANKL stimulation. Importantly, our TRAP staining results showed that numbers of TRAP-positive multinucleated osteoclasts (MNCs) formed reached their maximum at days three and four in both cases of RAW-D cells (Figure 1E and Figure S1C,D) and BMMs (Figure 1F and Figure S1E), but somewhat reduced at day five in RAW-D cell-derived osteoclasts, suggesting that mature osteoclasts, unless otherwise specified, were formed in vitro after three days of RANKL stimulation. Altogether, these results indicated that Rab11b was strongly up-regulated at a late stage of osteoclast differentiation.
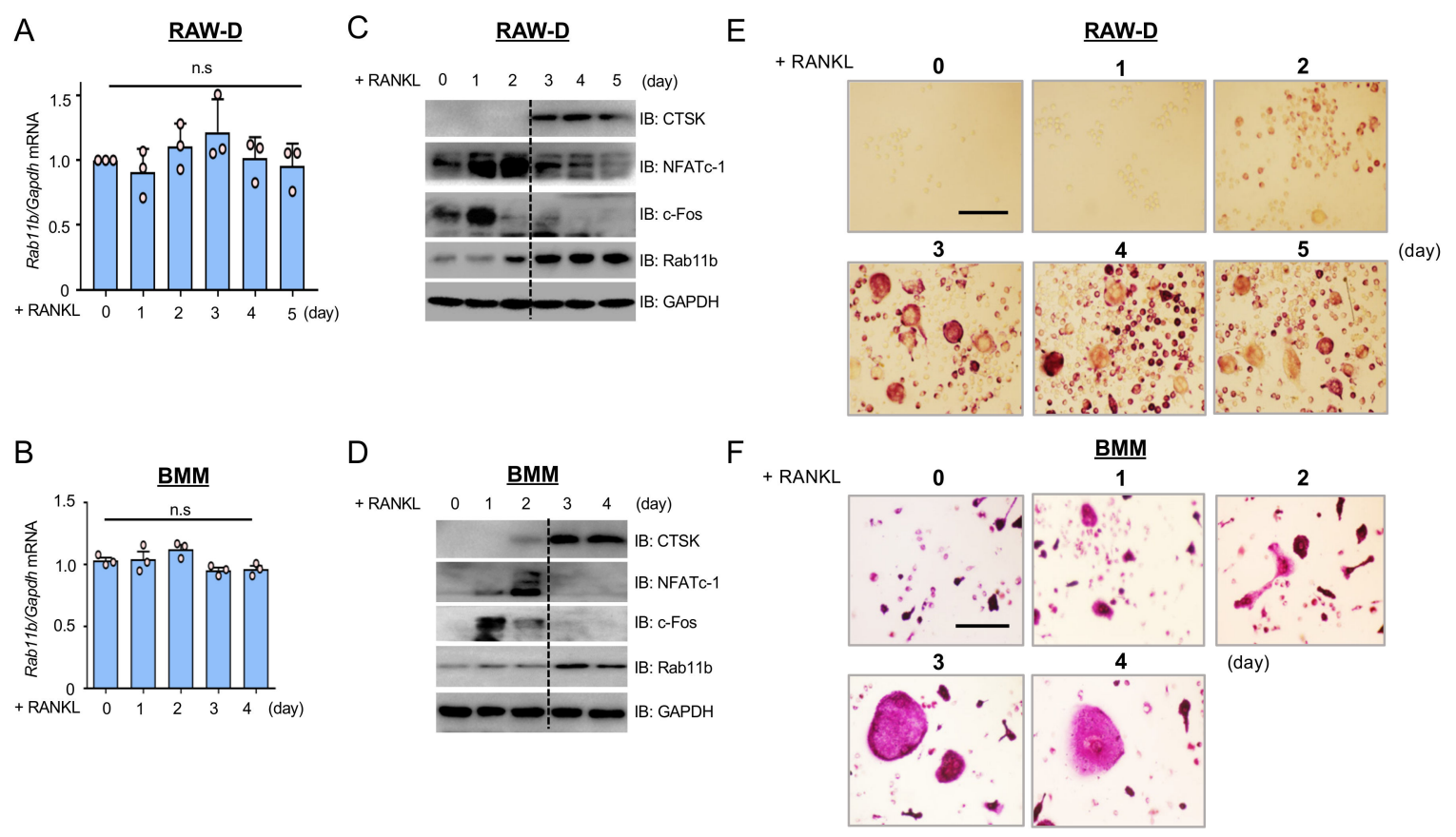

Figure 1. Rab11b upregulation at the late stage of osteoclastogenesis and the effect of RANKL (300 ng/mL) on osteoclast and monocyte/macrophage colony stimulating factor (M-CSF) (30 ng/mL) for a time course. (A,B) Total RNA was extracted, and cDNA was prepared from RAW-D cells or bone marrow-derived macrophages (BMMs) following a time course of RANKL ( $300 \mathrm{ng} / \mathrm{mL}$ ) stimulation. Rab11b mRNA expression levels were analyzed by quantitative Polymerase Chain Reaction (qPCR). Mean \pm SD of three independent repeats; n.s, nonsignificant (Student's $t$-test), $n=3$. (C,D) RAW-D cells or BMMs were treated with RANKL $(300 \mathrm{ng} / \mathrm{mL})$ over the indicated time course. Total expression levels of c-Fos, NFATc-1, CTSK, and Rab11b were assessed by immunoblotting and, GAPDH was used as a loading control. (E,F) TRAP-staining was carried out to assess osteoclast formation derived from RAW-D cells (E) and BMMs (F), following the indicated time course of RANKL stimulation. Bars $200 \mu \mathrm{m}$.

\subsection{Rab11b Silencing Markedly Enhances Osteoclastogenesis}

As above (Figure 1), Rab11b up-regulation was observed as mature osteoclasts were formed, it was surmised that Rab11b feasibly participated in inhibiting osteoclastogenesis. Thus, we first analyzed if osteoclastogenesis was affected by siRNA-mediated Rab11b suppression. Our data showed that Rab11b silencing promoted osteoclast formation in size (Figure 2A) and number (Figure 2B,C); more importantly, augmented the bone-resorbing activity (Figure 2D,E) in osteoclasts differentiated 
from RAW-D cells. The same effects were also found in BMM-derived osteoclasts (Figure S2A-D). In addition, Rab11b silencing enhanced expression levels of c-Fms, RANK, NFATc-1, and CTSK (Figure 2F) in osteoclasts differentiated from RAW-D cells (left panel) and BMMs (right panel). From these observations, we conjectured that Rab11b was a negative regulator of osteoclastogenesis.

Because Rab11a and b share $\sim 90 \%$ amino acid homology [13], it was crucial to confirm if Rab11b-targeting siRNA altered mRNA and protein levels of Rab11a. Intriguingly, our results showed that while Rab11b silencing slightly reduced, but insignificantly, mRNA levels of Rab11a (Figure S2E,F), it still increased expression levels of Rab11a (Figure S2G,H) in osteoclasts derived from both RAW-D cells and BMMs. These findings did clarify that Rab11b silencing rescued the expression level of Rab11a, suggesting that both homologs appeared to be functionally interrelated for the regulation of osteoclast differentiation.

\subsection{Rab11b Overexpression Significantly Abolishes Osteoclastogenesis}

In order to further elucidate the regressive role of Rab11b in osteoclastogenesis, we next evaluated if Rab11b overexpression altered osteoclast differentiation. We generated RAW-D cells stably expressing GFP, referred to as the control, and GFP-tagged Rab11b (GFP-Rab11b) with three different clones, abbreviated by \#1,\#2, and \#3, referred to as Rab11b overexpression. As we expectated, the bands showing GFP (Figure 2L, 6th panel from the top, 1st lane) and GFP-Rab11b (Figure 2L, 5th and 6th panel from the top, indicated by black arrows) expressions were detected in osteoclasts derived from RAW-D cells. Next, assessing osteoclast formation by TRAP staining, we found that Rab11b overexpression (i) diminished osteoclast size (Figure 2G), (ii) decreased the number of the TRAP-positive MNCs (Figure 2H), especially that of MNCs containing more than 10 nuclei (Figure 2I), and (iii) abolished the bone-resorbing ability of osteoclasts (Figure 2J,K) derived from RAW-D cells. In addition, expression levels of osteoclast markers, including c-Fms, RANK, and CTSK were markedly decreased whereas that of NFATc-1 was increased with respect to Rab11b overexpression (Figure 2L). Cumulatively, these results further postulated a critical role of Rab11b in negatively regulating osteoclastogenesis. By virtue of high structural and sequential homologies between Rab11a and b [13]; therefore, in order to ensure the authenticity of our results obtained so far, we tested if the primary antibody used to detect Rab11b was able to cross-react to Rab11a. As expected, our results showed that there was no cross-reaction of the primary antibody of Rab11b to Rab11a (Figure S2I, the top panel, red rectangle). Moreover, we observed that Rab11b overexpression declined expression level of Rab11a (Figure S2I, the top panel, purple rectangle), and reversely (Figure S2I, middle panel, yellow rectangles), further illuminating their interdependently functional roles in osteoclasts.

\subsection{Rab11b Overexpression Triggers $\mathrm{Ca}^{2+}$-Dependent NFATc-1 Stabilization}

Induction and activation of NFATc-1 [19,20] promoted CTSK gene transcription [21] during osteoclast differentiation. Notably, Rab11b overexpression weakened endogenous levels of c-Fms, RANK, and CTSK, but strengthened that of NFATc-1 in osteoclasts (Figure 2L). To unveil such an extraordinary phenomenon, we first assessed the dynamic modifications of NFATc-1 and CTSK over a time course of RANKL stimulation in RAW-D cells expressing GFP or GFP-Rab11b. Indeed, our data showed that Rab11b overexpression (i) decreased endogenous CTSK, but (ii) drastically enhanced that of NFATc-1 on day three (Figure 3A), relevant to our initial findings (Figure 2L). NFATc-1, a master regulator of osteoclast differentiation [22], could translocate into nuclei to transcriptionally promote osteoclast specific genes $[19,22]$. By nuclear/cytoplasmic fractionation, we found Rab11b overexpression enhanced cytosolic level, but decreased the nuclear level of NFATc-1 (Figure 3B). Similarly, to ensure our data consistency, we also investigated the effects of Rab11b silencing on regulating NFATc-1 and CTSK in osteoclasts derived from RAW-D cells. Indeed, Rab11b silencing markedly increased expression levels of these proteins in a time course of RANKL stimulation (Figure S3A), and accelerated nuclear translocation of NFATc-1 (Figure S3B), suggesting one potentiality of Rab11b overexpression-mediated NFATc-1 regulation independent of osteoclastogenesis signaling pathways induced by RANKL. 

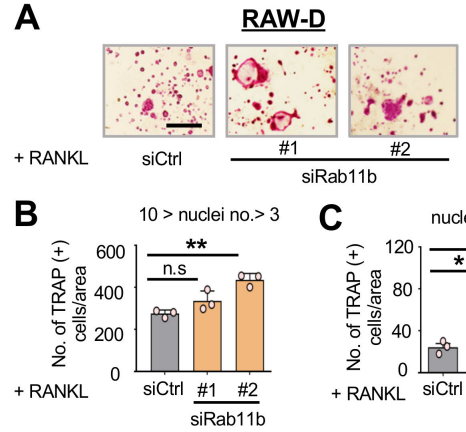

G
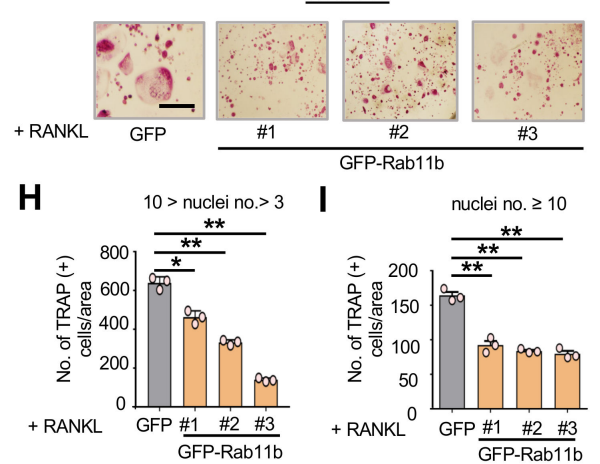

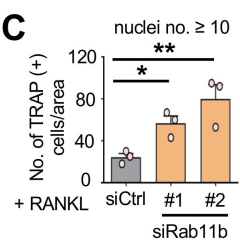

$\underline{\text { RAW-D }}$

$$
\text { I }
$$
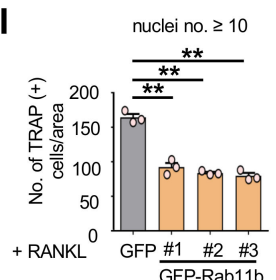

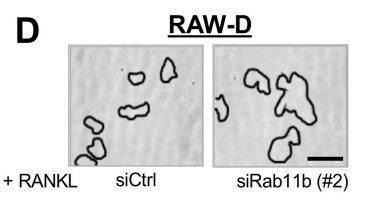

E

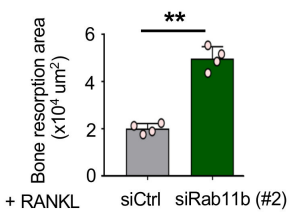

$\mathbf{J}$

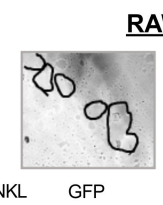

K

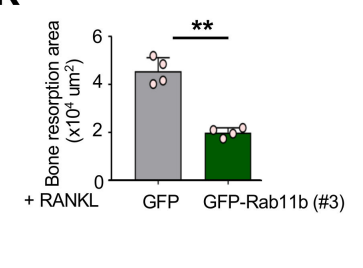

$\mathbf{F}$

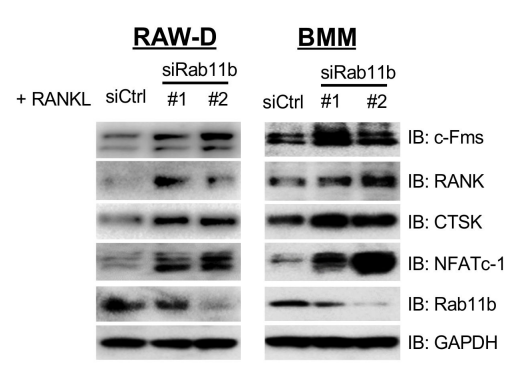

$\mathbf{L}$

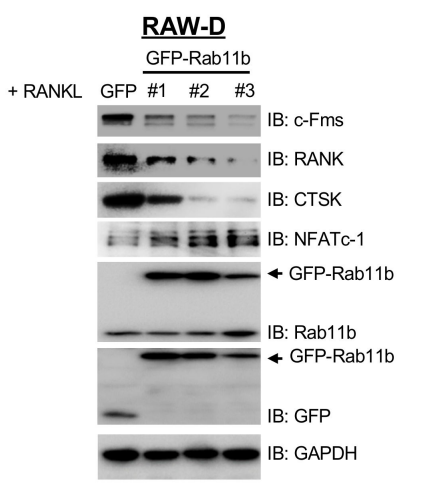

Figure 2. The effects of Rab11b knockdown and overexpression on osteoclastogenesis. (A) RAW-D cells were transfected with 10 pmol non-targeting siRNA (siCtrl) or two different types of Rab11b-specific siRNA (siRab11b \#1 and \#2) for $24 \mathrm{~h}$. Then, the cells were cultured for an additional 3 days with media containing RANKL (300 $\mathrm{ng} / \mathrm{mL}$ ). TRAP-staining was performed to evaluate osteoclast formation. Bars $200 \mu \mathrm{m}$. (B) The number of TRAP-positive osteoclasts simultaneously having more than 3 nuclei and less than 10 nuclei per viewing field was counted. ${ }^{* *} p<0.01 ;$ n.s, nonsignificant (Student's $t$-test); compared to the control. (C) The number of TRAP-positive osteoclasts having 10 or more nuclei per viewing field was counted. ${ }^{*} p<0.05 ;{ }^{* *} p<0.01$ (Student's $t$-test); compared to the control. Mean \pm SD of three independent repeats. (D) The images of the bone resorption area induced by RAW-D cell-derived osteoclasts transfected with siCtrl or siRab11b before seeded and cultured on the Osteo Assay StripWell upon RANKL (500 ng/mL) stimulation for 7 days. (E) The bone resorption area was measured and analyzed using Image J software. Mean \pm SD of triplicate samples. ${ }^{* *} p<0.01$ (Student's $t$-test), $n=3$. (F) After siRNA transfection for $24 \mathrm{~h}$, RAW-D cells and BMMs were differentiated into osteoclasts by being cultured with RANKL $(300 \mathrm{ng} / \mathrm{mL})$ and a combination of RANKL $(300 \mathrm{ng} / \mathrm{mL})+$ M-CSF (30 ng/mL), respectively, for an additional $72 \mathrm{~h}$. The total expression levels of c-Fms, RANK, NFATc-1, CTSK, and Rab11b were evaluated by immunoblotting, and GAPDH was used as a loading control. (F) The shown data were representative from two independent repeats. (G) RAW-D cells were transfected with retrovirus vectors either encoding Green Fluorescent Protein (GFP) as control or three different clones of GFP-Rab11b (abbreviated by \#1, \#2, and \#3). The cultured cells were incubated with RANKL ( $300 \mathrm{ng} / \mathrm{mL}$ ) for 3 days, and subsequently subjected to TRAP-staining analysis to assess the osteoclast formation. Bars $200 \mu \mathrm{m}$. (H) The number of TRAP-positive osteoclasts having simultaneously more than 3 and less than 10 nuclei was counted. Mean \pm SD of three independent repeats. ${ }^{*} p<0.05,{ }^{* *} p<0.01$ (Student's $t$-test); compared to the control. Bars $200 \mu \mathrm{m}$. (I) The number of TRAP-positive osteoclasts having 10 or more nuclei per viewing field was counted. Mean \pm SD of three independent repeats. ${ }^{* *} p<0.01$. (J) The bone-resorbing activities of the RAW-D-derived osteoclasts expressing GFP or GFP-Rab11b (clone \#3). The cells were seeded and cultured on the Osteo Assay Stripwell Plates upon RANKL $(500 \mathrm{ng} / \mathrm{mL})$ stimulation for 10 days. (K) The images of the bone-resorption area were measured and analyzed using Image J software. Mean $\pm \mathrm{SD}$ of the triplicate repeats. ${ }^{* *} p<0.01$ (Student's $t$-test) for the indicated comparisons. (L) The total expression levels of c-Fms, RANK, NFATc-1, CTSK, and Rab11b were evaluated by immunoblotting, and GAPDH was used as a loading control. The shown data were the representative from three independent repeats. 


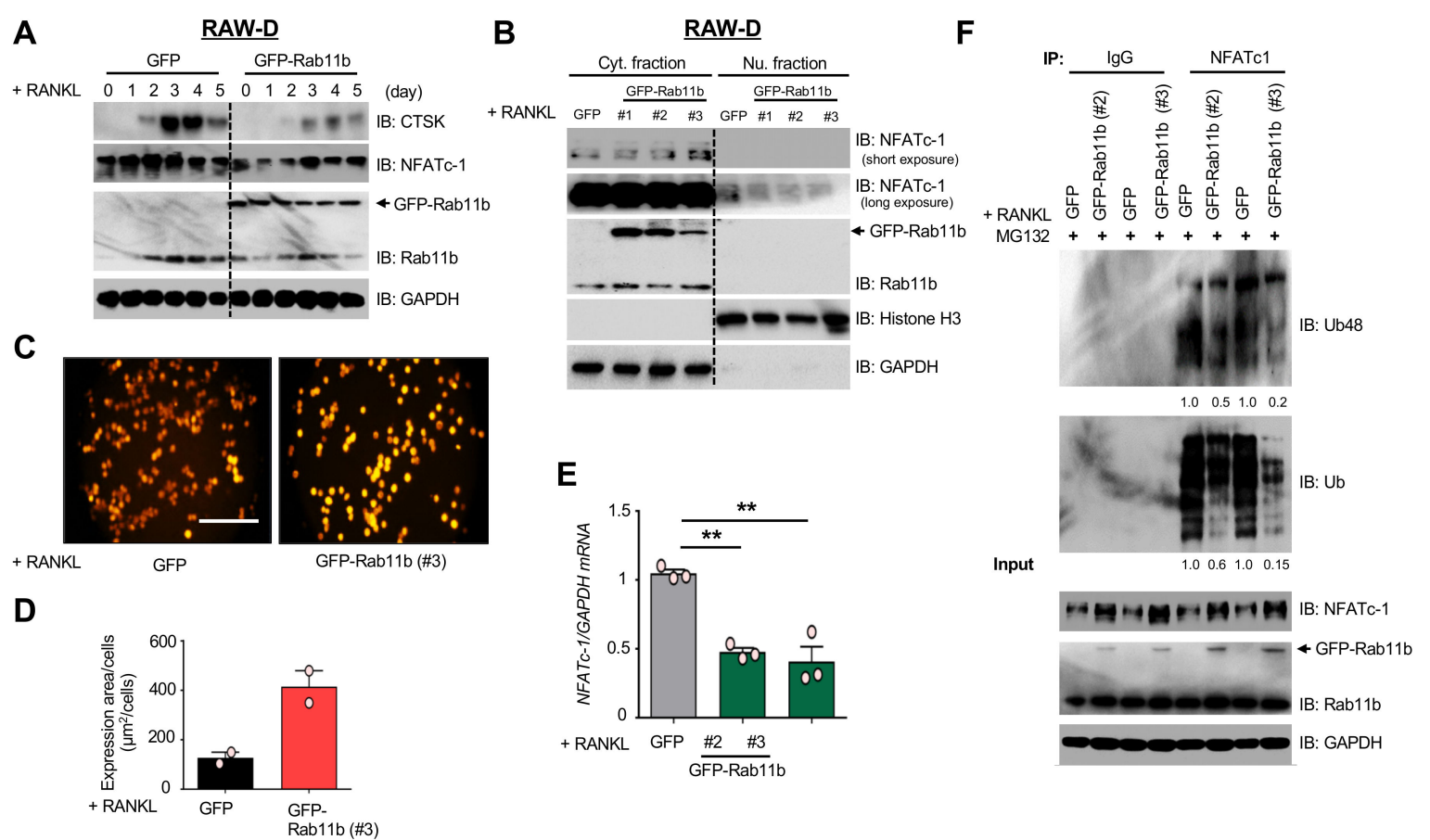

Figure 3. The effects of exogenous expression of Rab11b on NFATc-1 stabilization in response to $\left(\mathrm{Ca}^{2+}{ }_{\mathrm{i}}\right)$ elevation. (A) RAW-D cells expressing GFP or GFP-Rab11b (clone \#3) was incubated with RANKL (300 $\mathrm{ng} / \mathrm{mL}$ ) over a time course, and subsequently harvested. The cell lysates were subjected to SDS-PAGE followed by immunoblotting for the detection of CTSK, NFATc-1, Rab11b, and GAPDH was used as a loading control. The representative data were obtained from two independent repeats. (B) Cytosolic and nuclear fractions were prepared from the RAW-D cell-derived osteoclasts expressing GFP or one of three different clones of GFP-Rab11b (\#1, \#2, and \#3) upon RANKL (300 ng/mL) stimulation for 3 days. Cytosolic and nuclear fractions were subjected to SDS-PAGE and immunoblot analysis for detection of NFATc-1, Rab11b, and Histone H3 and GAPDH was used as the cytosolic and nuclear markers, respectively. The shown data were representative of two independent repeats. $(\mathrm{C})\left(\mathrm{Ca}^{2+}{ }_{\mathrm{i}}\right)$ oscillation in RAW-D cells expressing GFP or GFP-Rab11b (clone \#3) in response to RANKL stimulation. After RANKL (300 ng/mL) addition for 2 days, the cells were washed by the serum (-/-) media, and subsequently loaded with $1 \mu \mathrm{M}$ Cal-590 for $1 \mathrm{~h}$. The cells were then washed and analyzed by Olympus Uplsapo 10×. Bars $100 \mu \mathrm{m}$. (D) The $\left(\mathrm{Ca}^{2+}{ }_{\mathrm{i}}\right)$ fluorescent intensities were measured and analyzed by Image J software. Mean \pm SD of two independent repeats. (E) Quantitative Real Time-PCR (RT-PCR) analysis of NFATc-1 mRNA expression levels obtained from RAW-D cell-derived osteoclasts expressing GFP or one of two different clones of GFP-Rab11b (\#2 and \#3) after 3 days of RANKL $(300 \mathrm{ng} / \mathrm{mL})$ treatment. Mean $\pm \mathrm{SD}$ of triplicate repeats. ${ }^{* *} p<0.01$ (Student's $t$-test), compared to the control (GFP). (F) Osteoclasts differentiated from RAW-D cell expressing GFP or one of two different clones of GFP-Rab11b (\#2 and \#3) upon RANKL (300 ng/mL) for 3 days. The cells were incubated with MG132 (10 $\mu \mathrm{M}$ for $4 \mathrm{~h}$ ) before being lysed and subjected to anti-IgG or anti-NFATc-1 antibody IP followed by anti-Ub or anti-Ub48 with immunoblot analysis. The densitometer reading of polyubiquitinated NFATc-1 levels was measured underneath from 5th to 6th (for \#2) or from 7th to 8th (for \#3) lanes. The 5th or 7th lane was arbitrarily set as 1.0. The given results were obtained from two independent repeats.

Previous reports revealed that NFATc-1 deubiquitination was mediated by transient elevation of intracellular $\mathrm{Ca}^{2+}\left(\left(\mathrm{Ca}^{2+}{ }_{\mathrm{i}}\right)\right)$ [22-24]. We thus examined if cytosolic NFATc-1 accumulation by Rab11b overexpression initially resulted from the transient elevation of $\left(\mathrm{Ca}^{2+}{ }_{\mathrm{i}}\right)$ in osteoclasts. Our data showed that Rab11b overexpression markedly enhanced $\left(\mathrm{Ca}^{2+}{ }_{\mathrm{i}}\right)$ levels in RAW-D cells stimulated with RANKL for two days (Figure 3C,D). Furthermore, we tested transient $\left(\mathrm{Ca}^{2+}{ }_{\mathrm{i}}\right)$ oscillation by ionomycin, a selective $\mathrm{Ca}^{2+}$, efficacious in $\mathrm{Ca}^{2+}$ fluxes [25]. Our data showed that ionomycin treatment 
drastically elevated $\left(\mathrm{Ca}^{2+}{ }_{\mathrm{i}}\right)$ with respect to Rab11b overexpression (Figure S3C,D). More critically, Rab11b overexpression decreased mRNA levels of NFATc-1 (Figure 3E), but the increased endogenous level of NFATc-1, and abolished NFATc-1 polyubiquitination at lysine 48 (Figure 3F), suggesting a novel mechanism of Rab11b overexpression-mediated regulation of $\left(\mathrm{Ca}^{2+}{ }_{\mathrm{i}}\right)$-dependent NFATc-1 stabilization, following RANKL stimulation.

2.5. Rab11b Modulates NFATc-1 Signaling Cascades in the RAW-D Cells and BMMs upon Stimulation with RANKL and M-CSF, Respectively

Due to Rab11b-mediated declination of endogenous levels of c-Fms and RANK (Figure 2L), we, therefore, tested how NFATc-1 upstream signaling cascades would be altered with respect to Rab11b overexpression. Indeed, our results indicated that Rab11b overexpression caused the phosphorylation levels of $\mathrm{p} 38$, I $\mathrm{KB} \alpha$, Akt, and Erk to be strongly decreased while that of JNK was indistinguishable (Figure 4A). Furthermore, we earlier showed that Rab11b silencing activated NFATc-1 (Figure 2F and Figure S3A,B), we, therefore, examined the effects of Rab11b silencing on the osteoclastogenic signaling cascades in BMMs over a time course of M-CSF stimulation. Indeed, our results showed that phosphorylation levels of p38, Akt, and Erk were significantly increased in Rab11b-silenced BMMs (Figure 4B). Together, these data elucidated the stimulatory effects of Rab11b silencing on modulating osteoclastogenic signaling cascades, thereby promoting osteoclast differentiation.

\section{A}

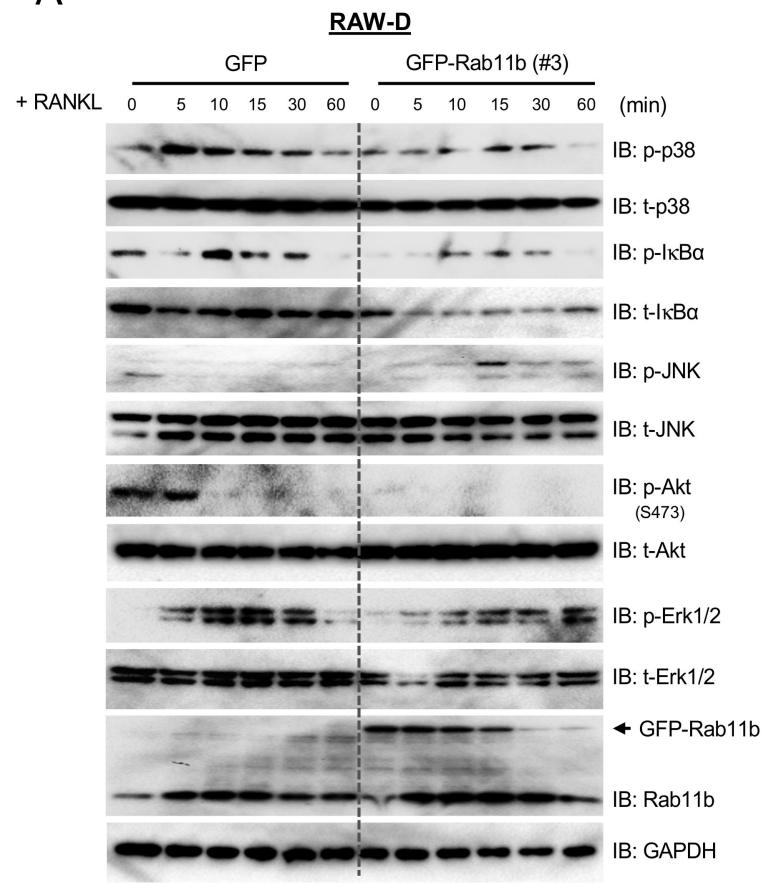

B

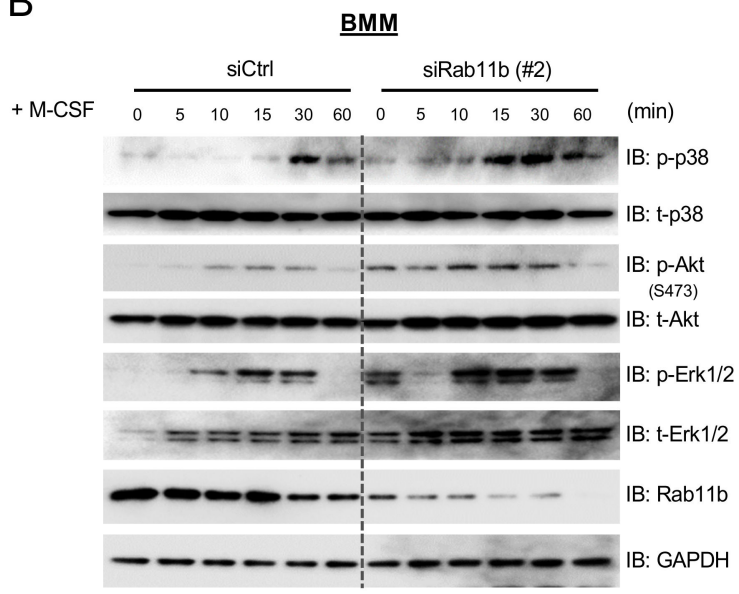

Figure 4. The effects of Rab11b on NFATc-1 upstream signaling cascades in macrophages stimulated by RANKL and M-CSF, respectively. (A) RAW-D cells expressing GFP or GFP-Rab11b (clone \#3) were incubated with serum (-/-) culture media in the absence of RANKL. After RANKL (300 ng/mL) supplementation, the cells were incubated for the indicated times, and subsequently collected. The cell lysates were subjected to SDS-PAGE followed by immunoblotting for the detection of total expression levels of p-p38, t-p38, p-IкB $\alpha, t-I \kappa B \alpha, p-J N K, t-J N K, p-A k t$ (S473), t-Akt, p-Erk 1/2, t-Erk 1/2, Rab11b, and GAPDH was used as a loading control. The shown data were obtained from two independent repeats. (B) BMMs transfected with non-targeting siRNA (siCtrl) or Rab11b siRNA (siRab11b, type 2 ) were pre-incubated with serum (-/-) culture media in the absence of M-CSF. Then, the cells were incubated with M-CSF (50 ng/mL) for the indicated periods, and subsequently harvested. The cell lysates were subjected to SDS-PAGE followed by immunoblotting for the detection of p-p38, t-p38, p-Akt (S473), t-Akt, p-Erk 1/2, t-Erk 1/2, Rab11b, and GAPDH was used as a loading control. 
2.6. GFP-Rab11b Localized in Early and Late Endosomes, Golgi Complex, and Endoplasmic Reticulum Causes the Enlargement of Early and Late Endosomes in RAW-D Cells and Osteoclasts

An earlier report identified Rab11b required for the transport of internalized transferrin to the plasma membrane in CHO cells localizes in endocytic recycling compartments (ERCs) [26]; however, little is known about the subcellular localization of Rab11b in RAW-D cells and osteoclasts. To address it, co-localization of the stably expressed GFP-Rab11b was examined with several organelle-specific marker proteins such as Rab5 (early endosomes, EEs), Rab7 (late endosomes, LEs), GM130 (Golgi complex), LAMP1 (lysosomes), and KDEL (endoplasmic reticulum, ER). Our results revealed that Rab11b was co-localized with Rab5, Rab7, GM130, and KDEL, but not LAMP1 in both RAW-D cells (Figure S4A-C), and RAW-D-derived osteoclasts (Figure 5A-C). Of note, we observed that Rab11b overexpression caused stronger intensity of (i) Rab5-positive fluorescence and enlarged Rab5-positive EEs in RAW-D cells (Figure S4D) and osteoclasts (Figure 5D), and (ii) those of Rab7-positive LEs in RAW-D cells (Figure S4E) and osteoclasts (Figure 5E), but not (iii) those of GM130-positive Golgi complex in RAW-D cells (Figure S4F), and in osteoclasts (Figure 5F). From these observations, we conjectured that size-based enlargement of EEs and LEs possibly resulted from the accumulation of internalized cargos in EEs and LEs in both RAW-D cells and osteoclasts differentiated from RAW-D cells, suggesting that, regardless of RANKL stimulation, Rab11b overexpression was sufficient for such a morphological alteration in EEs and LEs.

\subsection{Rab11b-Mediated Augmentation of Lysosomal Function Regulates Endogenous Turnovers of c-Fms and RANK in Osteoclasts}

Previous studies comprising ours have highlighted the functional importance of lysosomes in osteoclast differentiation [17,27-29]; hence, we tested if Rab11b alteration could affect the function of the lysosomal system in osteoclasts. Experimentally, our results showed Rab11b overexpression resulted in markedly elevated expression levels of lysosomal LAMP1, and Cathepsins B and D (Figure 6A) whereas its suppression insignificantly altered patterns of such lysosomal markers (Figure S5A), prompting that Rab11b was engaged in regulating lysosomal functions in osteoclasts. Because Rab11b overexpression decreased expression levels of c-Fms and RANK, we examined if lysosomes were involved in c-Fms and RANK degradation in osteoclasts. To address this question, we analyzed the effects of lysosomal inhibition by a lysosomal blocker, chloroquine (CLQ), in osteoclasts. Our results showed lysosomal inhibition increased endogenous levels of c-Fms and RANK, but not Rab11b, in doseand time-dependent manners (Figure 6B,C); more importantly, no significant alteration of mRNA levels of c-Fms (Figure 6D) and RANK (Figure 6E) was observed between each group treated with or without CLQ over a time course in osteoclasts expressing GFP or GFP-Rab11b. In addition, we found that Rab11b overexpression substantially declined levels of c-Fms (Figure 6D) and RANK (Figure 6E) mRNAs. Since proteasome complex is thought to be responsible for cellular proteolysis [30,31], we used MG132, a peptide aldehyde proteasome inhibitor, to analyze the effects of proteasome inhibition on endogenous levels of c-Fms and RANK. Amazingly, no marked alternation of patterns of c-Fms and RANK were found in response to MG132 treatment (ranging from 0 to $20 \mu \mathrm{M}$ ) (Figure S5B). These data indicated that regulation of c-Fms and RANK by lysosomal inhibition, but not by proteasome inhibition, was likely due to their post-translational regulation in osteoclasts.

To further explore the detailed mechanism of lysosome-mediated proteolysis of c-Fms and RANK, it was essential to inhibit new protein synthesis by cycloheximide (CHX). We initially determined an optimal concentration of CHX used for the subsequent experiments. Our results showed an inhibitory effect of CHX $(20 \mu \mathrm{g} / \mathrm{mL})$ on endogenous levels of RANK and c-Fms was stabilized after $3 \mathrm{~h}$ treatment (Figure S5C) in osteoclasts expressing GFP and GFP-Rab11b. Treatment of (CHX + CLQ) combination markedly increased endogenous levels of c-Fms and RANK as compared to those treated with CHX alone (Figure 6F), regardless of Rab11b overexpression. Moreover, no significant alteration of patterns of c-Fms and RANK were found in each group treated with CHX-alone or with (CHX + MG132) combination (Figure S5D). In addition, no cytotoxic effects were found in all cases treated with the 
drug(s) (Figure S5E-F). Taken together, these data emphasized an important role of lysosomes in regressing osteoclast differentiation through mediating proteolysis of c-Fms and RANK.
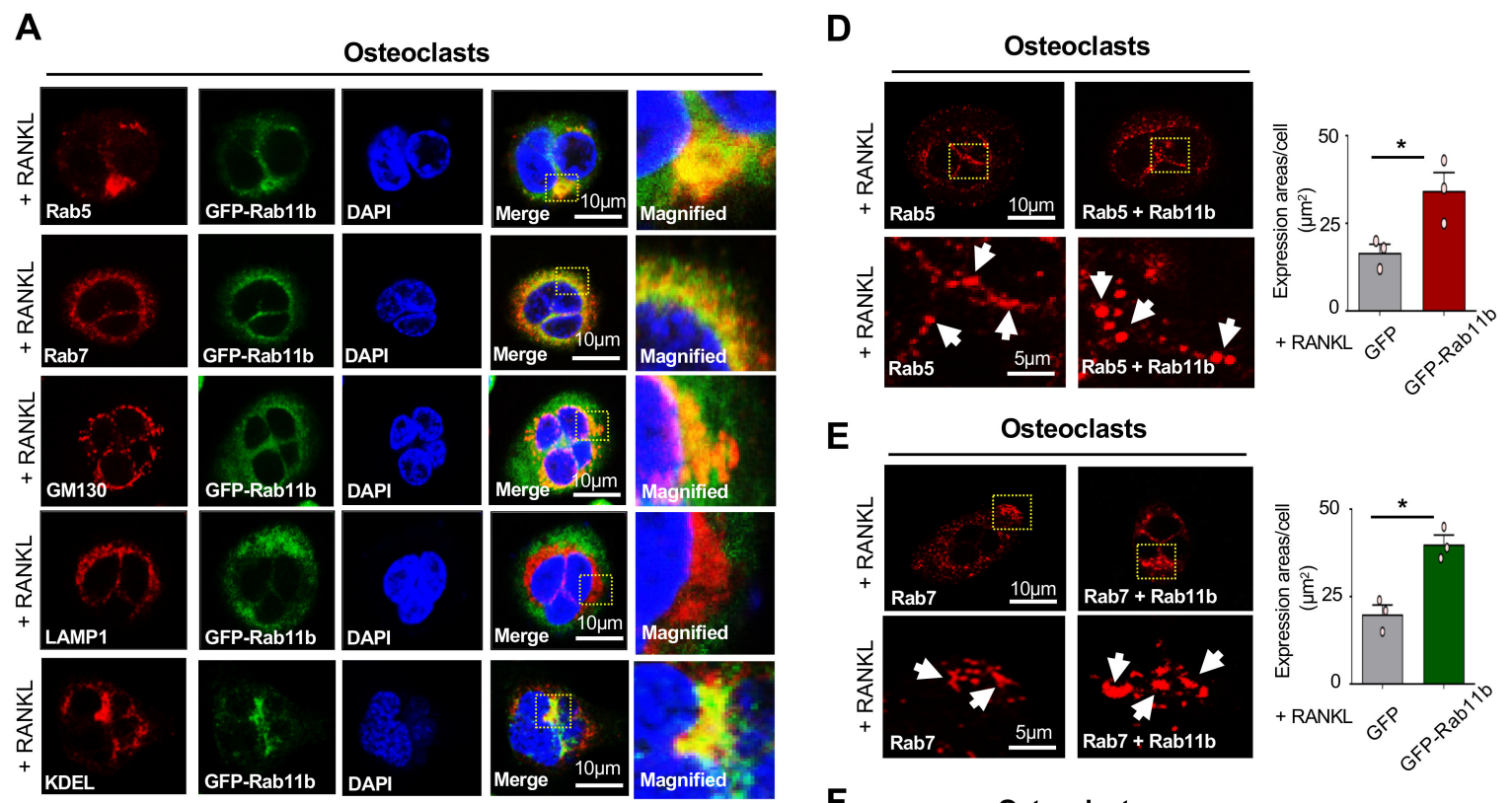

B

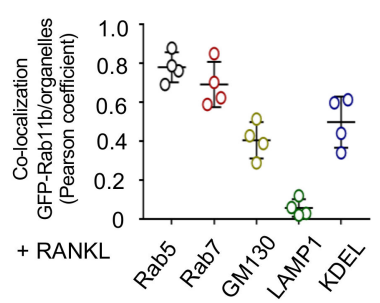

C

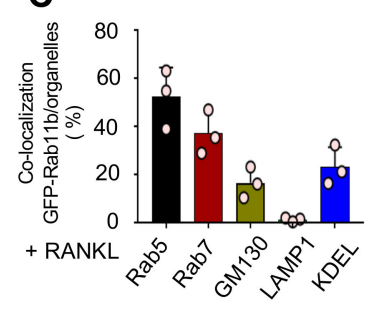

$\mathbf{F}$

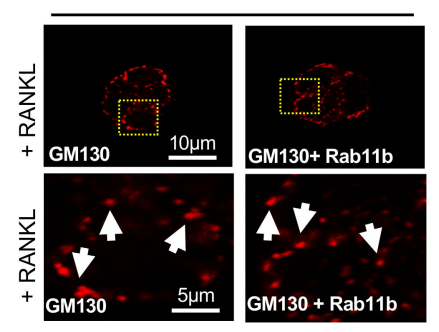

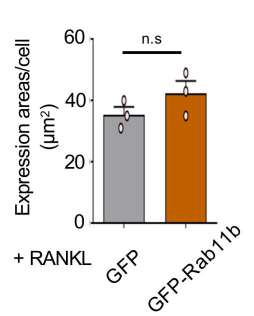

Figure 5. Subcellular localization of Rab11b in RAW-D cell-derived osteoclasts, and the effects of Rab11b overexpression on size-based modification of organelles. (A) The osteoclasts derived from RAW-D cells stably expressing GFP-Rab11b (clone \#1) (green) were seeded on cover glasses with fixation and permeabilization of $0.2 \%$ Triton X-100 in PBS, and subsequently reacted with one of the antibodies against Rab5, Rab7, MG130, LAMP1, or KDEL (red, as indicated) that are specific markers for early endosomes, late endosomes, Golgi complex, lysosomes, or endoplasmic reticulum, respectively. Osteoclast DNA was stained with 4',6-diamidino-2-phenylindole (DAPI) (blue). Specific regions of interest within a field are shown by yellow boxes in a magnified form on the left-hand top of each image it was taken from. Specific regions of interest within a field, designated by yellow boxes, are magnified on the right side of each image it was taken from. Mean $\pm \mathrm{SD}$ of three independent repeats. Scale bar: $10 \mu \mathrm{m}$. (B,C) GFP-Rab11b co-localization with Rab5, Rab7, MG130, LAMP1 and KDEL was determined and evaluated by Pearson coefficient (B) or by color threshold analysis (C) using Fiji/ImageJ on at least 4 cells. (D-F) RAW-D cells stably expressing GFP or GFP-Rab11b were fixed and permeabilized with $0.2 \%$ Triton X-100, and stained with the specific antibodies to detect (D) Rab5 (red), (E) Rab7 (red), and (F) MG130 (red). The images were captured by confocal laser microscopy. The particle size was measured by $\left(\mu \mathrm{m}^{2}\right)$ using ImageJ (shown on the right side). Mean $\pm \mathrm{SD}$ of at least four independent repeats. ${ }^{*} p<0.05$, n.s., nonsignificant (Student's $t$-test). 

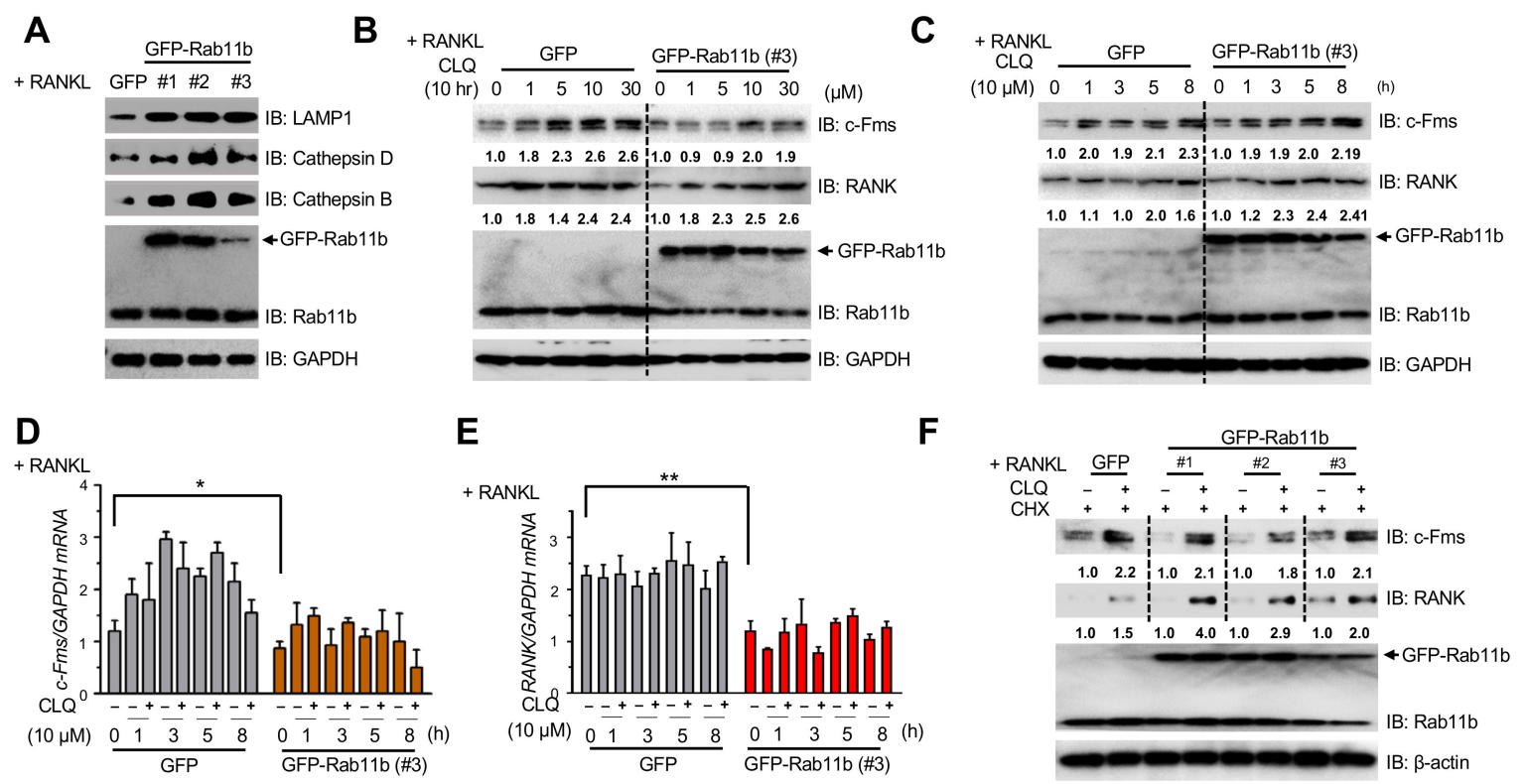

Figure 6. The effects of CLQ-mediated lysosomal function on endogenous levels of c-Fms and RANK in RAW-D cell-derived osteoclasts. (A) RAW-D cells expressing GFP or one of three different types of GFP-Rab11b (\#1, \#2, and \#3) were treated with RANKL (300 ng/mL) for 3 days. The immunoblotting analysis of endogenous levels of LAMP1, a specific lysosomal receptor, and two lysosomal enzymes, Cathepsins B and D was done. GAPDH was used as a loading control. The shown data was representative of two independent repeats. (B) RAW-D cells expressing GFP or GFP-Rab11b (type \#3) was pre-treated with RANKL (300 ng/mL) for 3 days before incubation with CLQ $(0,1,5,10$, and $30 \mu \mathrm{M}$ ) for $10 \mathrm{~h}$. The WB analysis of endogenous levels of c-Fms, RANK, and Rab11b was done. GAPDH was used as a loading control. The shown data was representative of two independent repeats. (C) RAW-D cells expressing GFP or GFP-Rab11b (type \#3) were pre-treated with RANKL (300 ng/mL) for 3 days before incubation with CLQ $(10 \mu \mathrm{M})$ over a time course $(0,1,3,5$, and $8 \mathrm{~h})$. The WB analysis of endogenous levels of c-Fms, RANK, and Rab11b was done. GAPDH was used as a loading control. $(\mathrm{D}, \mathrm{E})$ Total RNA was extracted, and cDNA was prepared from osteoclasts differentiated from RAW-D cells expressing GFP or GFP-Rab11b (type \#3), following RANKL (300 ng/mL) stimulation for 3 days. Expression levels of c-Fms (D) and RANK (E) mRNAs were analyzed by qRT-PCR. Mean \pm SD of two independent repeats. ${ }^{*} p<0.05,{ }^{* *} p<0.01$ (Student's $t$-test). (F) RAW-D cells expressing GFP or one of three different types of GFP-Rab11b (\#1, \#2, and \#3) were pre-treated with RANKL (300 ng/mL) for 3 days. The cells were subsequently treated with CHX $(20 \mu \mathrm{g} / \mathrm{mL})$ and simultaneously with or without CLQ $(10 \mu \mathrm{M})$ for $5 \mathrm{~h}$. The WB analysis of endogenous levels of c-Fms, RANK, and Rab11b was done. $\beta$-actin was used as a loading control. The shown data was the representative of two independent repeats.

\subsection{Rab11b Overexpression Promoted Lysosome-Mediated Degradation of c-Fms and RANK Receptors in Osteoclasts}

As above, Rab11b localization was observed in early and late endosomes. One of the well-characterized transport routes of lysosome-degraded cargos, including surface receptors, is sequentially coated, sorted, and processed by early and late endosomes [32-34]. Consequently, we speculated that Rab11b promoted the delivery of c-Fms and RANK receptors from early, late endosomes to lysosomes in osteoclasts. We first evaluated how Rab11b alteration affected surface levels of c-Fms and RANK receptors in osteoclasts. Our results showed Rab11b overexpression strongly decreased surface levels of c-Fms and RANK receptors (Figure 7A,B) whereas its silencing rescued their surface levels (Figure 7C,D), unveiling a suppressive effect of Rab11b on surface levels of c-Fms and RANK receptors in osteoclasts. Next, we analyzed if Rab11b promoted lysosome-mediated degradation of c-Fms and RANK receptors. By pre-treating osteoclasts with a combination of CHX $(20 \mu \mathrm{g} / \mathrm{mL})$ 
and MG132 $(10 \mu \mathrm{M})$, we found that endogenous levels of c-Fms and RANK were rapidly decreased in response to Rab11b overexpression (Figure $7 \mathrm{E}-\mathrm{G}$ ), and no cytotoxic effects on osteoclast viability were observed in all treated cases (Figure $7 \mathrm{H}$ ). Altogether, these results illuminated a novel function of Rab11b as an accelerator of the lysosome-mediated degradation of c-Fms and RANK receptors in osteoclasts.

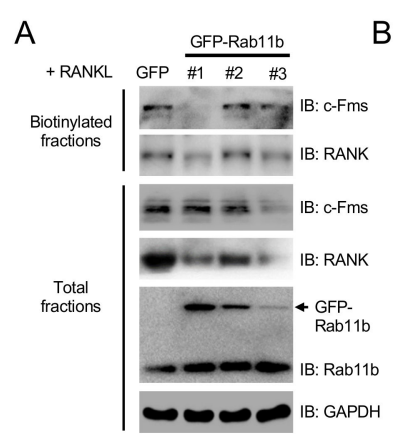

C

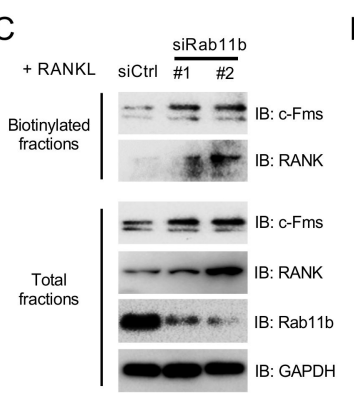

B
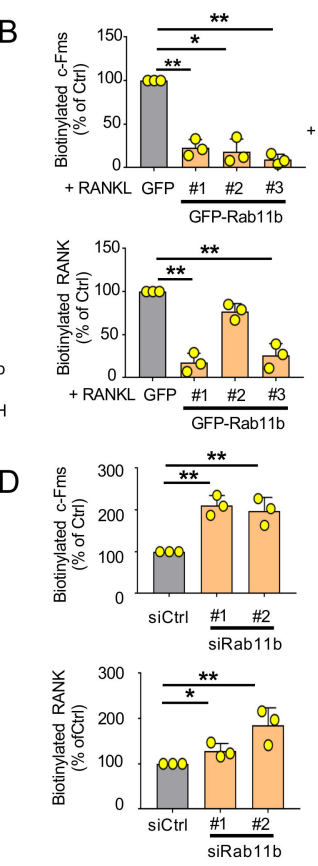

E \begin{tabular}{lllllllll}
\multicolumn{4}{c}{ GFP } & & \multicolumn{4}{c}{ GFP-Rab11b (\#1) } \\
\hline 0 & 1 & 3 & 5 & 0 & 1 & 3 & 5
\end{tabular}

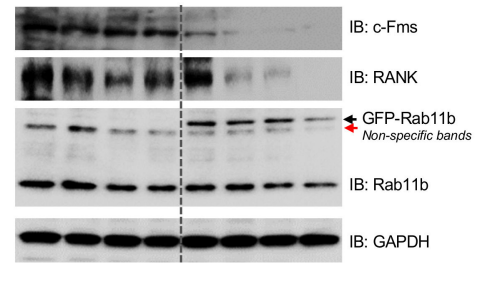

G

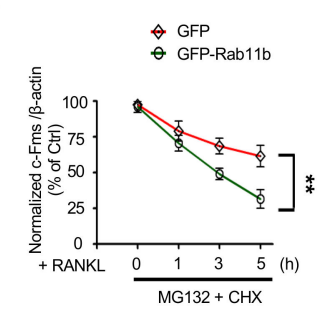

$\mathrm{H}$

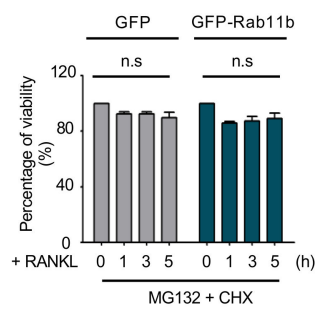

Figure 7. The effects of Rab11b overexpression on lysosome-mediated proteolysis of c-Fms and RANK receptors in osteoclasts. (A) RAW-D cells expressing GFP or three different types of GFP-Rab11b (\#1, \#2, and \#3) were stimulated with RANKL ( $300 \mathrm{ng} / \mathrm{mL}$ ) for 3 days. The cells were fractionated into two separate fractions including biotinylated fraction and total fraction as the protocol indicated in the "Material and Methods" section. The surface and total levels of c-Fms and RANK were evaluated by biotinylation pulldown and immunoblotting, respectively. (B) The densitometry quantification of bands indicating surface expression of c-Fms and RANK receptors was shown in the presence of GFP-Rab11b as a percentage of control (GFP). Mean \pm SD of three independent repeats; * $p<0.05$, ** $p<0.01$ (Student's $t$-test). (C) RAW-D cells were transfected with scrambled siRNA (siCtrl) or one of two different Rab11b siRNAs (Rab11b siRNA \#1, Rab11b siRNA \#2), followed by RANKL (300 ng/mL) stimulation for 3 days. The cells were fractionated into two separate fractions including biotinylated fraction and total fraction as the above protocol. The surface and total levels of c-Fms and RANK were evaluated by biotinylation pulldown and immunoblotting, respectively. (D) The densitometry quantification of bands indicating surface expression of c-Fms and RANK receptors was shown in the presence of Rab11b siRNAs as a percentage of control (siCtrl). Mean \pm SD of three independent repeats; ${ }^{*} p<0.05,{ }^{* *} p<0.01$ (Student's $t$-test). (E) RAW-D cells expressing GFP or GFP-Rab11b (\#1) were stimulated with RANKL $(300 \mathrm{ng} / \mathrm{mL}$ ) for 3 days, followed by treatment with a combination of MG132 $(20 \mu \mathrm{M})$ and CHX $(20 \mu \mathrm{g} / \mathrm{mL})$ for $0,1,3$, and $5 \mathrm{~h}$. Total levels of c-Fms and RANK were evaluated by immunoblotting. $(\mathrm{F}, \mathrm{G})$ The densitometry quantification of bands indicating total expression of c-Fms (F) and RANK (G) was shown as a percentage of the 1st lane for the GFP group referred to as the control (Ctrl), and the 5th lane for the GFP-Rab11b group referred to as the control (Ctrl). Mean \pm SD of three independent repeats. ${ }^{* *} p<0.01,{ }^{* * *} p<0.001$ (Student's $t$-test). (H) Cell viability was assessed by the cellular ATP content measurement using the Cell Titer Glo Assay system. After stimulated with RANKL ( $300 \mathrm{ng} / \mathrm{mL}$ ) for 3 days, RAW-D cells were co-treated with CHX $(20 \mu \mathrm{g} / \mathrm{mL})$ and CLQ (10 $\mu \mathrm{M})$ over a time course. The values were the average of triplicate determinations with SD indicated by error bars. n.s., nonsignificant (Student's $t$-test). The experiments were repeated thrice. 


\section{Discussion}

In this study, we have provided compelling results elucidating that Rab11b was up-regulated at a late stage of osteoclast differentiation and maturation. Notably, Rab11b mRNA level (Figure 1A,B) was insignificantly varied during osteoclast differentiation, suggesting it was highly possible that Rab11b could be stabilized by post-translational modification, for instance, by deubiquitinases. Nonetheless, searching for potential candidates serving Rab11b deubiquitination has been under investigation. Here, by loss and gain of Rab11b expression experiments, Rab11b, for our first time, has been identified as a negative regulator of osteoclast differentiation. Indeed, Rab11b silenced an increased number of multinucleated and giant osteoclasts, enhanced bone resorbing activity, and strengthened endogenous levels of osteoclast markers (Figure 2 and Figure S2) while Rab11b overexpression inhibited such properties of osteoclasts (Figure 2). Intriguingly, we found that Rab11b overexpression triggered $\mathrm{Ca}^{2+}$-dependent NFATc-1 deubiquitination and stabilization (Figure 3).

Previous reports have disclosed the physiological role of transient elevation of intracellular $\mathrm{Ca}^{2+}$ in sequential events of dephosphorylation, deubiquitination, stabilization, and nucleus translocation of NFATc-1 and that it was crucial for promoting transcriptional processes of osteoclastogenic genes such as CTSK, TRAP, and MMP9 [21,35,36]. Yet, it was surprising that, by Rab11b overexpression, the cytoplasmic NFATc-1 level was increased while nuclear NFATc-1 level was decreased in osteoclasts (Figure 3B). These data raised questions of how Rab11b overexpression could accumulate NFATc-1 in the cytosol, and what the potent mechanism of nuclear NFATc-1 translocation regulation was. Importantly, Rab11b overexpression declined surface abundance of c-Fms and RANK receptors (Figure 7A), thereby attenuating NFATc-1 upstream signaling cascades (Figure 4), raising one feasibility that nuclear NFATc-1 translocation could be controlled by unknown factors that were regulated by certain NFATc- 1 upstream signaling pathways dependent on c-Fms/RANK receptor activation. Resultantly, our future works are to identify such important factors so as to further understand underlying molecular mechanisms of NFATc-1 regulation during osteoclast differentiation.

Rab11b was mainly localized in early and late endosomes in RAW-D cells (Figure S4A) and osteoclasts (Figure 5A); furthermore, Rab11b overexpression caused size-based enlargement of early and late endosomes, consistent with our initial findings, in which Rab11b was up-regulated at a late stage of osteoclast differentiation. Furthermore, it was possible that Rab11b up-regulation accelerated cargo internalization into early and late endosomes. Surprisingly, an earlier study revealed CRISPR/Cas9-based depletion of either Rab11a or b caused enlargement of early endosomes, and enhancement of late endosomal and lysosomal activities in non-polarized HeLa cells [37], contradictory to our observations in osteoclasts, indicating a broad spectrum of Rab11b functional features in human cells. Although it was clarified that Rab11b did not localize in lysosomes (Figure 5), Rab11b overexpression up-regulated LAMP1, and Cathepsins B and D in osteoclasts (Figure 6A), proposing that Rab11b was possibly linked to lysosomal functions. Besides, we also observed Rab11b localization in Golgi complex, and ER in both RAW-D cells and osteoclasts; nonetheless, how it was functionally linked to cargo delivery amongst these organelles in osteoclasts has been completely obscure. One of our speculations is that Rab11b is probably engaged in regulating other osteoclast receptors, required for osteoclastogenesis regulation, by the direct- or indirect-transport of intracellular receptors to cell surfaces, distinct from the transport route of c-Fms and RANK surface receptors via the axis of early endosomes-late endosomes-lysosomes. Calcitonin receptor (CTR), for instance, directly binds to calcitonin, a calcium-lowering hypocalcemic hormone secreted by the thyroidal C $[38,39]$, was demonstrated to trigger osteoclast retraction, abnormally transfigured osteoclast morphology [40], and finally inhibition of bone resorption [41,42]. Astonishingly, CTR expression was enhanced before the formation of RANKL-induced multinucleated osteoclasts $[43,44]$. Therefore, one of our future research goals would be examining if Rab11 is functionally involved in regulation of surface abundance of CTR or CTR-like receptor (CLR) during osteoclast differentiation.

Albeit that lysosomal involvement in osteoclast differentiation regulation has been highlighted [17,28,29], a lysosomal role for proteolytic cleavage of c-Fms and RANK has not been previously reported. 
In our study, using a specific lysosomal inhibitor, we revealed a crucial role of lysosomes in c-Fms and RANK proteolysis, regardless of Rab11b overexpression (Figure 5B,C,F), suggesting a natural characteristic of lysosomes as a suppressive regulator of osteoclast differentiation. To prove lysosomal vitality to proteolysis of c-Fms and RANK, we also evaluated the effect of MG132-mediated inhibition of proteasome complex upon proteolytic cleavage of c-Fms and RANK. Different from our observations in lysosomes, proteasome complex inhibition did not alter endogenous levels of c-Fms and RANK, suggesting that neither c-Fms nor RANK could undergo post-translational modifications by ubiquitin-proteasome complex. These data further strengthened our observational theory of a unique lysosomal function on proteolysis of c-Fms and RANK in osteoclasts. It is worth noting that reduction of the surface abundance of c-Fms and RANK receptors results in weakening osteoclastogenic signal transduction, thereby debilitating osteoclastogenesis [6,45-48]. Interestingly, our data revealed Rab11b overexpression decreased surface abundance of c-Fms and RANK receptors (Figure 7A,B) via ameliorating proteolysis of c-Fms and RANK (Figure 7E), further substantiating a suppressive role of Rab11b in osteoclast differentiation.

Because the individual Rab11 family members have not been broadly studied, it has been unclear that Rab11a and b have overlapping or distinct functions in osteoclasts. Whether Rab25, found to regulate integrin expression in colonic epithelial cells [15], is present in osteoclasts has been controversial. Interestingly, our recent study elucidated that Rab11a played an important role in negatively regulating osteoclast differentiation [18]; we therefore attempted to investigate the functional conjunction between Rab11a and b, using loss and gain of Rab11b expression experiments. As predicted, siRNA-mediated Rab11b suppression rescued the Rab11a expression in osteoclasts derived from RAW-D cells (Figure S2G) and BMMs (Figure S2H). Furthermore, Rab11b overexpression weakened the expression level of Rab11a, and reversely (Figure S2I), suggests that both homologs (i) are functionally interdependent of each other and (ii) are negative regulators of osteoclast differentiation. In addition, we also hypothesized that other Rab GTPases might have functionally interconnected and cooperated with each other for the regulation of osteoclastogenesis. To Rab11b, it might be either Rab11a, unknown factors, or both (Figure 8).

In conclusion, our study, the first report to shed light on the critical role of Rab11b as a negative regulator of osteoclast differentiation via directing and transporting c-Fms and RANK receptors via a selective axis of early endosomes-late endosomes-lysosomes for proteolytic degradation, subsequently abolishing osteoclastogenesis, eventually stabilizing the osteoclastic bone resorption phase. The study of how inhibitory regulators worked on osteoclast differentiation was beyond our knowledge of macrophagic features and osteoclastic bone resorption. Finally, our novel findings may contribute to facilitate the establishment of either efficacious drugs, potential therapeutic methods, or both, for the treatment of macrophage-caused inflammatory diseases related to bone loss, consisting of osteoporosis, Paget's disease, periodontitis, and rheumatoid arthritis, all of which are characterized by excessive bone resorption by gain of function in osteoclasts [49-52]. Over the past decade, the introduction of target biologic therapy having significantly improved the clinical outcomes for patients with bone-related diseases abovementioned, is mediated by RANKL blockade [50] or by targeting pro-inflammatory cytokines [53] in order to prevent osteoclast differentiation, which subsequently reduces bone erosion. However, our study has comprehensibly elucidated a novel insight of how the bone-resorbing capacity of osteoclasts was inhibited by a mechanism underlying Rab11b-mediated lysosomal degradation of c-Fms and RANK surface receptors, raising one probability of the drug development program for treatment of the bone-related diseases caused by gain of function in osteoclasts aimed at either activating lysosomal function in combination with RANKL blockade in pre-osteoclasts or upregulating Rab11 in both pre-osteoclasts and osteoclasts. 


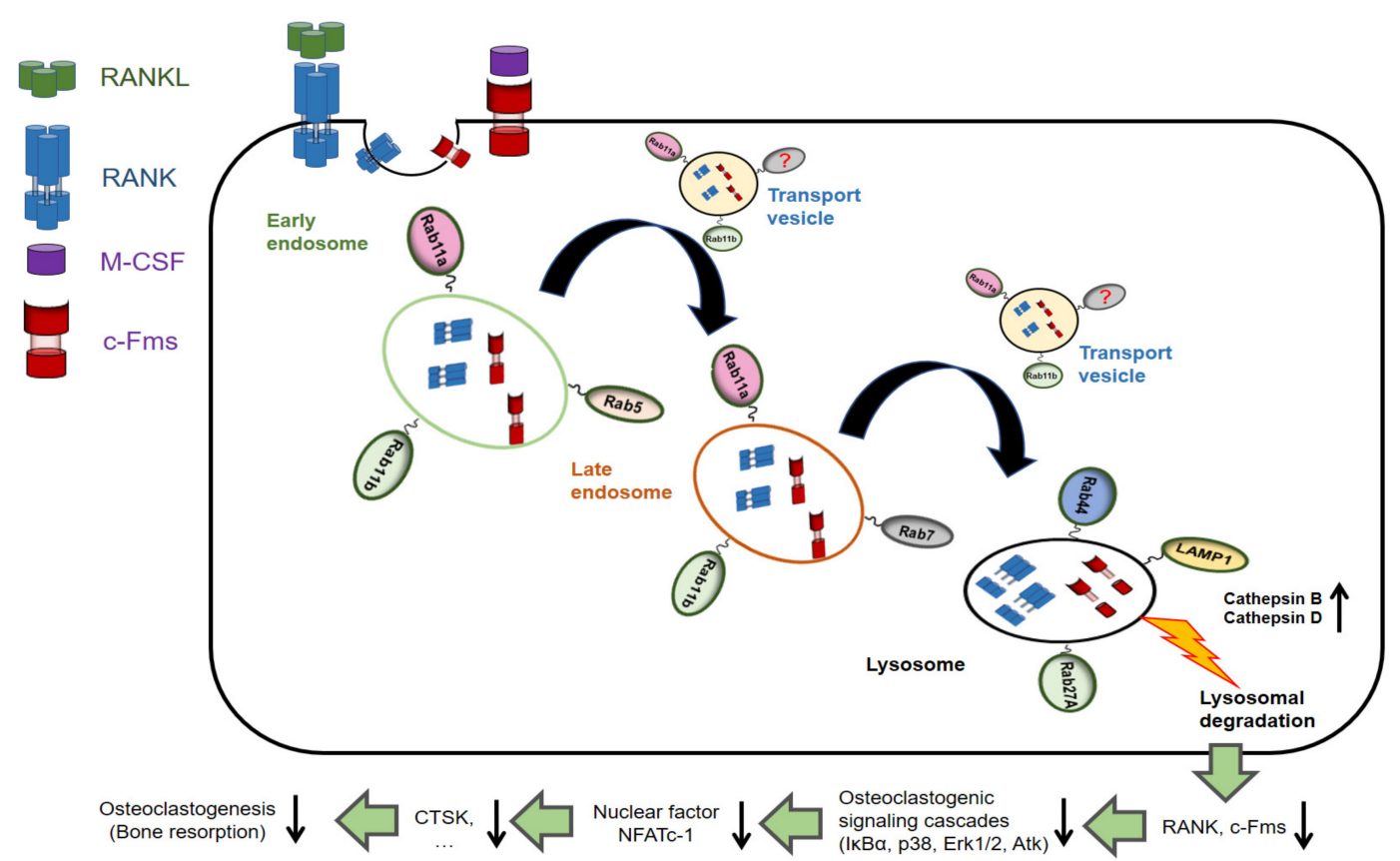

Figure 8. The model epitomizing the findings of our current works. c-Fms and RANK receptors were early internalized into early endosomes, and transported to late endosomes by the transport vesicles, which was subsequently fused with lysosomes for proteolysis of c-Fms and RANK surface receptors. Both homologs, Rab11a and b, upregulated at a late stage of osteoclast differentiation dictated the transport of c-Fms and RANK surface receptors to the lysosome via the axis of early and late endosomes-lysosomes. Rab11-mediated lysosomal proteolysis of c-Fms and RANK receptors sequentially weakened the osteoclastogenic signaling cascades, inhibited the nuclear translocation of the transcription factor, NFATc-1, reduced the expression level of CTSK, and eventually abolished osteoclastogenesis (bone resorption).

\section{Materials and Methods}

\subsection{Antibodies and Reagents}

Isolation and purification of recombinant RANKL was prepared by the protocols described heretofore [54]. M-CSF was purchased from Kyowa Kogyo (Tokyo, Japan). Rabbit polyclonal anti-Cathepsins B and D, K antibodies were purified as the protocol described previously [55]. The following antibodies were used in this study: rat monoclonal anti-LAMP1 (Cat. No. 553792, BD Biosciences, NJ, USA), rabbit polyclonal anti-c-Fms (Cat. No. sc-692, Santa Cruz, CA, USA), mouse monoclonal anti-c-Fms (\#G1019) (Santa Cruz Biotechnology, Santa Cruz), mouse monoclonal anti-c-Fos (\#1019) (Santa Cruz Biotechnology, Santa Cruz, CA 95060, USA), mouse monoclonal anti-NFATc-1 (Cat. No. sc-7294, Santa Cruz, CA, USA), mouse monoclonal anti-RANK (NBP2-247-2, Novus Biologicals Europe, Abingdon, UK), rabbit polyclonal anti-GFP (Green Fluorescent Protein) (Medical \& Biological Laboratories Co., LTD., Nagoya, Japan), rabbit polyclonal anti-Rab11a (\#2413), and rabbit polyclonal anti-Rab11b (\#2414), rabbit monoclonal anti-Rab5 (\#3547), rabbit monoclonal anti-Rab7 (\#9367) (Cell Signaling, Danvers, MA, USA), mouse monoclonal GM130 (Cat. no. 610823) (BD, Biosciences, Franklin Lakes, NJ, USA), mouse monoclonal anti-KDEL (Cat. no. ADI-SPA-827) (Enzo Life Sciences, Farmingdale, NY, USA), rabbit monoclonal anti-phospho-p38 (Cat. no. 4511S, Thr180/Tyr182), rabbit polyclonal anti-p38 MAPK (Cat. no. 9212S), rabbit monoclonal anti-phospho-IkB $\alpha$ (Cat. no. 2859S, Ser32), rabbit monoclonal anti-IкB $\alpha$ (Cat. no. 4812S), rabbit polyclonal anti-phospho-JNK (Cat. no. 9251S, Thr183/Tyr185), rabbit polyclonal anti-JNK (Cat. no. 9252S), rabbit monoclonal anti-phospho-Akt (protein kinase B) (Cat. no. 4060S, Ser473), rabbit monoclonal anti-Akt (Cat. no. 4691S), rabbit polyclonal anti-phospho-Erk1/2 (Cat. no. 9101S, Thr202/Tyr204), rabbit polyclonal 
anti-Erk1/2(Cat. no. 9102S), mouse monoclonal anti-ubiquitin (\#8017) Santa Cruz Biotechnology, Santa Cruz, CA 95060), rabbit polyclonal K48-linked ubiquitin (\#4289S) (Cell, Signaling, Danvers, MA, USA), rabbit monoclonal anti-GAPDH (Cat. no. 2118S), Alexa Fluor 488 goat anti-rabbit IgG, Alexa Fluor 488 goat anti-mouse IgG, and Alexa Fluor 594 goat anti-rat IgG (Cell Signaling Technology Danvers, MA, USA). DAPI (4',6-diamidino-2-phenylindole) and other reagents purchased are as follow: Cal-590TM AM (COSMO bio Co., Ltd., Japan) and Ionomycin (WAKO, Osaka, Japan). Other reagents were purchased from Sigma-Aldrich (Tokyo, Japan). The Osteo Assay Stripwell Plate was purchased from Corning, Inc. (Corning, NY, USA).

\subsection{Cell Culture}

A murine monocytic cell line (also known as RAW-D) gifted from Toshio Kukita (Kyushu University, Japan) [56,57] was cultured in minimum essential medium $\alpha(\mathrm{MEM} \alpha)$ (Wako Pure Chemicals, Osaka, Japan) supplemented with 10\% fetal bovine serum (FBS), penicillin $(100 \mathrm{U} / \mathrm{mL})$ and streptomycin $(100 \mathrm{mg} / \mathrm{mL})$. For osteoclast differentiation from RAW-D cells, RAW-D cells were cultured in MEM $\alpha$ with RANKL ( $300 \mathrm{ng} / \mathrm{mL}$ ) for 3 days. Bone marrow-derived macrophages (BMMs) were isolated from the femurs and tibias of 5-week-old male C57BL/6J mice (SLC, Shimizu Laboratory, Japan) as described previously [54] through flushing the bone marrow cavity, and subsequently cultured in MEM $\alpha$ with M-CSF $(50 \mathrm{ng} / \mathrm{mL})$ at $37{ }^{\circ} \mathrm{C}$ in $5 \% \mathrm{CO}_{2}$. One day later, the non-adherent cells were collected and cultured in new MEM $\alpha$ with M-CSF ( $30 \mathrm{ng} / \mathrm{mL}$ ). On day 3 of culturing, the adherent cells, referred to as BMMs, were refreshed with new MEM $\alpha$ with M-CSF $(30 \mathrm{ng} / \mathrm{mL})$. For osteoclast differentiation from BMMs, BMMs were cultured in MEM $\alpha$ with both M-CSF $(30 \mathrm{ng} / \mathrm{mL})$ and RANKL ( $300 \mathrm{ng} / \mathrm{mL})$ for 3 days.

\subsection{Quantitative Real-Time Polymerase Chain Reaction (RT-PCR) Analysis}

For real-time PCR analysis, Trizol (Molecular Research Center, Cincinnati, OH, USA) was used for RNA extraction and purification from cultured cells. Following which, $0.1 \mu \mathrm{g}$ of total RNA was reverse transcribed for cDNA synthesis using an iScript cDNA Synthesis Kit (Bio-Rad, Hercules, CA, USA). Quantitative real-time PCR was done using MJ Mini (BioRad), according to the manufacturer's instructions. The primer sets used are indicated in Table 1.

Table 1. List of sequences of DNA primers designed and used for quantitative real-time PCR.

\begin{tabular}{ccc}
\hline Primer $(\mathbf{N a m e})$ & Forward Sequence $\left(\mathbf{5}^{\prime} \rightarrow \mathbf{3}^{\prime}\right)$ & Reverse \\
\hline Rab11a & ACGTCATCTCAGGGCAGTTC & TTGGCTTGTTCTCAGTGGTG \\
Rab11b & AGAAGCTAAAAGCCCCTTGC & CAACTGGCCAGCGCGGAAAG \\
NFATc-1 & TCATCCTGTCCAACACCAAA & TCACCCTGGTGTTCTTCCTC \\
c-Fms & TTGGACTGGCTAGGGACATC & GGTTCAGACCAAGCGAGAAG \\
RANK & CTTGGACACCTGGAATGAAGAAG & AGGGCCTTGCCTGCATC-3 \\
GAPDH & ACCACAGTCCATGCCATCAC & TCCACCACCCTGTTGCTGTA \\
\hline
\end{tabular}

\subsection{Immunoblot Analysis}

Cells were seeded and grown to confluence in either $60 \mathrm{~mm}$ dishes or $10 \mathrm{~cm}$ dishes, followed by appropriate treatments detailed in the result section. Cells were lysed by RIPA buffer ( $50 \mathrm{mM}$ Tris- $\mathrm{HCl}$ (pH 8.0), $1 \%$ Nonidet P-40, $0.5 \%$ sodium deoxycholate, $0.1 \%$ SDS, $150 \mathrm{mM} \mathrm{NaCl}$ ) supplemented with proteinase inhibitor cocktail (Sigma-Aldrich, Tokyo, Japan), put on ice for $30 \mathrm{~min}$, and subsequently centrifuged for $20 \mathrm{~min}$ at 15,000 rpm. The protein concentrations were subsequently determined by Bio-Rad assay (Thermo Pierce, Rockford, IL, USA) according to the manufacturer's guidance. The cell lysates ranging from 15 to $50 \mu \mathrm{g}$ were run on 10\% SDS-PAGE electrophoresis gels, and then transferred to polyvinylidene fluoride (PVDF) membranes by a wet-transfer method. The blots were blocked in Tris-buffered saline containing $0.05 \%$ Tween 20 and $5 \%$ skim milk for $1-1.5 \mathrm{~h}$ at room temperature (RT), and subsequently probed with various antibodies $(1 / 1000)$ at $4{ }^{\circ} \mathrm{C}$ overnight. After being washed, the blots were incubated with appropriate horseradish peroxidase (HRP)-conjugated secondary antibodies 
(GE Healthcare). Blots were eventually reacted with ECL substrate (Millipore, Burlington, MA, USA). The immunoreactive bands were observed with a ChemiDoc MP Imaging System (Bio-Rad, Hercules, CA, USA). To confirm equal amounts of loaded samples, the blots were incubated with either an anti-GAPDH-HRP antibody for $1 \mathrm{~h}$ or an anti-Actin antibody $\left(4^{\circ} \mathrm{C}\right.$, overnight), and subsequently incubated with rabbit HRP-conjugated secondary antibody.

\subsection{RNA Interference}

RAW-D cells or BMMs cultured in $60 \mathrm{~mm}$ dishes $\left(2 \times 10^{5}\right.$ cells/dish $)$ for protein analysis or $\left(1 \times 10^{5}\right.$ cells/dish) for RNA analysis, were transfected with 10 pmol of one of two independent duplex siRNAs (Rab11b) covering the targeted sequences: 5'-GGGACGACGAGUACGAUUACCUAUU-3' (Rab11b siRNA \#1), or 5'-AAUAGGUAAUCGUACUCGUCGUCCC-3' (Rab11b siRNA \#2) (Invitrogen Custom Primers, Invitrogen, Carlsbad, CA, USA), or with 10 pmol of non-targeting siControl (siCtrl) (Stealth RNAi siRNA Negative Control, Invitrogen, Carlsbad, CA, USA) diluted in Opti-MEM I (Life Technologies) with Lipofectamine RNAiMAX transfection reagent (Invitrogen, Carlsbad, CA, USA) at $37^{\circ} \mathrm{C}$ overnight, according to the manufacturer's instructions. At the 1st day of post-transfection, the cells were replaced and cultured with new culture media containing RANKL ( $300 \mathrm{ng} / \mathrm{mL}$ ) for 3 days. The knockdown efficacy was assessed by qPCR, immunoblotting, and TRAP staining where appropriate. Only for bone resorption analysis, after siRNA transfection, the cells were cultured with culture media supplemented with RANKL ( $500 \mathrm{ng} / \mathrm{mL}$ ) for 7-10 days.

\subsection{TRAP Staining}

Cells were fixed with $4 \%$ paraformaldehyde (PFA) at RT for $1 \mathrm{~h}$, and subsequently treated with $0.2 \%$ Triton X-100 in PBS at RT for 5 min. Ultimately, cells were stained for TRAP solution including $0.01 \%$ naphthol AS-MX phosphate (Sigma-Aldrich, Tokyo, Japan) and 0.06\% fast red violet LB salt (Sigma-Aldrich, Tokyo, Japan) in the presence of $50 \mathrm{mM}$ sodium tartrate and $50 \mathrm{mM}$ sodium acetate ( $\mathrm{pH}$ 5.0). TRAP-positive multinuclear cells were counted under a light microscope, and the images were photographed by the Olympus FSX100 microscope. The multinuclear cells harboring three or more nuclei were considered as mature osteoclasts.

\subsection{Immunocytochemistry}

The cells seeded and grown on glass coverslips were fixed with 4.0\% PFA in PBS for $1 \mathrm{~h}$ at RT. After washing with PBS, the fixed cells were permeabilized with $0.1 \%$ Triton X-100 in PBS for 10 min. The cells were incubated sequentially with $10 \%$ normal goat serum for $30 \mathrm{~min}$ and with primary antibodies at $4{ }^{\circ} \mathrm{C}$ overnight. The cells were washed, and stained with secondary antibodies, Alexa Fluor 594 goat anti-rat IgG or Alexa Fluor 594 goat anti-rabbit IgG (Cell Signaling Technology, Danvers, MA, USA). Ultimately, nuclear staining with DAPI (Invitrogen Carlsbad, CA, USA) was carried out. The samples were visualized using a laser-scanning confocal imaging system (LSM 780 META; Carl Zeiss, AG, Jena, Germany).

\subsection{Bone Resorption Assay}

The bone resorption assay was done by the method described previously. The bone-resorbing activity of osteoclasts derived from RAW-D cells and BMMs upon M-CSF ( $30 \mathrm{ng} / \mathrm{mL}$ ) and RANKL ( $500 \mathrm{ng} / \mathrm{mL}$ ) stimulation for 7-10 days was determined using the Osteo Assay Stripwell Plates. The images of the bone resorption area were photographed by Floid cell imaging station (Thermo Fisher, Waltham, MA, USA) and analyzed by Image J software.

\subsection{Retrovirus Construction and Expression of Mouse Rab11b in RAW-D Cells}

Retrovirus construction and expression of Rab11b were carried out as the methods described heretofore [16]. Briefly, the full-length cDNAs of mouse Rab11b were generated by PCR method 
employing cDNA originated from bone marrow macrophages (BMMs) simultaneously stimulated with M-CSF and RANKL for $72 \mathrm{~h}$. The primers were used for GFP (for in-fusion) forward: 5'-GGACG AGCTGTACAAGGGCACCCGCGACGACGAGTAC-3' and reverse: 5'-CTACCCGGTAGAATTCTT AGATGTTCTGACAGCACTGC-3'. Then, the cDNA(s) were amplified using Prime STAR GXL DNA polymerase (Takara, Tokyo) with 40 cycles at which denaturation at $94{ }^{\circ} \mathrm{C}$ for $10 \mathrm{~s}$, annealing at $62{ }^{\circ} \mathrm{C}$ for $30 \mathrm{~s}$, and extension at $72{ }^{\circ} \mathrm{C}$ for $3 \mathrm{~min}$ for each cycle. To generate GFP-Rab11b fusion protein, the amplified fragments were fused with a linearized pMSCVpuro-GFP, gifted by Kosei Ito (Nagasaki University, Japan), using In-Fusion cloning kit (Clontech, Mountain View, CA, USA). pMSCVpuro-GFP was also used as a control vector. GFP or GFP-Rab11b vectors were transfected into HEK293T cells by using Lipofectamine 2000 (Life Technologies, Gaithersburg, MD, USA), according to the manufacturer's instructions. After incubation at $37^{\circ} \mathrm{C}$ in $5 \% \mathrm{CO}_{2}$ for $48 \mathrm{~h}$, the supernatants composed of viruses were collected and subsequently infected into RAW-D cells. The cells were cloned by puromycin $(5 \mu \mathrm{g} / \mathrm{mL})$ diluted in MEM $\alpha$ supplemented with $10 \%$ FBS, penicillin $(100 \mathrm{U} / \mathrm{mL})$, and streptomycin $(100 \mathrm{mg} / \mathrm{mL})$, and the medium was refreshed every 3 days. After 2 weeks of culturing, puromycin-resistant cells were obtained and referred to as RAW-D cells expressing GFP or GFP-Rab11b.

\subsection{CellTiter-Glo Viability Assay (CTG)}

Cytotoxicity evaluation was carried out using the CellTiter-Glo Luminescent Cell Viability Assay Kit (Promega, Madison, WI, USA), according to the manufacturer's protocol. Furthermore, $5 \times 10^{3}$ cells/well were seeded and grown in 95 flat-bottomed well plates. The plate was incubated at $37^{\circ} \mathrm{C}$ in $5 \% \mathrm{CO}_{2}$ for $24 \mathrm{~h}$ prior to RANKL ( $300 \mathrm{ng} / \mathrm{mL}$ ) supplementation. The plates were incubated for 3 days in $5 \% \mathrm{CO}_{2}$, then simultaneously added with cycloheximide (CHX) $(20 \mu \mathrm{g} / \mathrm{mL})$ with or without chloroquine CLQ $(10 \mu \mathrm{M})$, or with or without MG132 $(20 \mu \mathrm{M})$ to each well, and subsequently incubated for the designated periods before being quenched with CellTiter-Glo (Promega, Madison, WI, USA, $50 \mu \mathrm{L} /$ well), then centrifuged at $1000 \mathrm{rpm}$ for $1 \mathrm{~min}$ and incubated at RT for $15 \mathrm{~min}$. Luminescence was measured with a plate reader (Molecular Devices, San Jose, CA, USA).

\subsection{Nuclear/Cytoplasmic Fractionation}

The subcellular fractionation was carried out using NE-PER nuclear and cytoplasmic extraction kit (Thermo Scientific). Cells were harvested by using cell scrapers, lysed with CER I containing protease inhibitors, and then incubated on ice for $15 \mathrm{~min}$. Permeabilized cells were subsequently added with ice-cold CER II, vortexed for $10 \mathrm{~s}$, incubated on ice for $1 \mathrm{~min}$, and centrifuged at $500 \times g$ for $10 \mathrm{~min}$ at $4{ }^{\circ} \mathrm{C}$. The pellets were put on ice, and the supernatants were further centrifuged at $16,000 \times g$ for $20 \mathrm{~min}$ at $4{ }^{\circ} \mathrm{C}$. Following which, $16,000 \times g$ supernatants were collected and referred to as the cytoplasmic fraction. After being washed twice with ice-cold PBS, the pellets were suspended in ice-cold NER containing protease inhibitors, and subsequently vortexed on the highest speed for $1 \mathrm{~h}$ at $4{ }^{\circ} \mathrm{C}$. After centrifugation at $16,000 \times \mathrm{g}$ for $15 \mathrm{~min}$ at $4{ }^{\circ} \mathrm{C}$, the supernatants were collected and referred to as the nuclear fraction.

\subsection{In Vitro Ubiquitination Assay}

RAW-D cells $\left(1 \times 10^{6}\right.$ cells) were seeded and grown in $10 \mathrm{~cm}$ culture dishes, then stimulated with RANKL ( $300 \mathrm{ng} / \mathrm{mL}$ ). After 3 days of culture, the cells were washed twice by cold PBS, scraped by a scraper. Furthermore, $0.8-1.0 \mathrm{mg}$ of whole cell lysates prepared by lysis in $150 \mathrm{mM} \mathrm{NaCl}, 1 \%$ Nonidet P-40, 1\% deoxycholate, 0.1\% SDS, $50 \mathrm{mM}$ Tris (pH 7.5), $1 \mathrm{mM}$ PMSF, $25 \mathrm{mM} \mathrm{NaF}$, and protease inhibitors, was added with mouse-IgG or NFATc1 antibody on the rotator at $4{ }^{\circ} \mathrm{C}$ for $1-2 \mathrm{~h}$. The immune complexes were incubated with protein-G beads overnight, preblocked with $10 \%$ bovine serum albumin. The immunoprecipitates were washed thrice with the same lysis buffer, and twice with IP buffer. The immunoprecipitates were mixed with $6 \times$ Laemmli dye, boiled at $60{ }^{\circ} \mathrm{C}$ for $20 \mathrm{~min}$, and loaded on SDS-PAGE gels. After transfer, the blots were blocked with the $5 \%$ skim milk dissolved in TBS-T for 1-2 $\mathrm{h}$, and probed with anti-ubiquitin antibody or anti-K48-ubiquitin antibody at $4{ }^{\circ} \mathrm{C}$ 
overnight. The blots were washed thrice with TBS-T, probed with secondary antibody sequentially, and developed.

\subsection{Surface Biotinylation Assay}

RAW-D cells $\left(5 \times 10^{5}\right.$ cells) were seeded and grown in a $10 \mathrm{~cm}$ dish for 3 days upon RANKL (300 ng/mL) stimulation. Cells were washed twice with cold PBS, and subsequently incubated for 1 $\mathrm{h}$ at $4{ }^{\circ} \mathrm{C}$ with $3.0 \mathrm{mg} / \mathrm{mL}$ Sulfo-NHS-SS-Biotin (Pierce) in DPBS+. Cell dishes were gently rinsed in $100 \mathrm{mM}$ glycine $(10 \mathrm{~min}, 3 \times)$, and subsequently in $20 \mathrm{mM}$ glycine $(10 \mathrm{~min}, 3 \times)$, both in DPBS+. Cells were harvested, and lysed by the buffer LB3 encompassing $50 \mathrm{mM}$ Tris/ $\mathrm{HCl}(\mathrm{pH} 7.4), 150 \mathrm{mM} \mathrm{NaCl}$, $1 \mathrm{mM}$ EDTA, $1 \%(\mathrm{w} / \mathrm{v})$ Triton X-100, and protease inhibitor. The cell lysates were gently rotated for $1 \mathrm{~h}$ at $4{ }^{\circ} \mathrm{C}$. The cell lysates were subsequently gently rotated by a rotator overnight at $4{ }^{\circ} \mathrm{C}$ with 40 $\mu \mathrm{L}$ Ultra Link Immobilized NeutrAvidin protein (Pierce). Followed by the incubation, beads were washed $1 \times$ with lysis buffer LB3, $2 \times$ with LB2 (LB3 not composed of protease inhibitor), $2 \times$ with SWS containing $0.1 \%$ Triton X-100 in PBS (pH 7.4), $350 \mathrm{mM} \mathrm{NaCl}$ and $1 \mathrm{mM} \mathrm{EDTA}$, and $1 \times$ with LB1 (LB2 not composed of $1 \%(w / v)$ Triton X-100). Then, the beads were completely mixed with $6 \times$ Laemmli dye and boiled for $5 \mathrm{~min}$ before being loaded on SDS-PAGE gels.

\subsection{Intracellular $\mathrm{Ca}^{2+}$ Measurement in Cell Populations}

RAW-D cells $\left(5 \times 10^{4}\right.$ cells) expressing GFP or GFP-Rab11b were seeded and grown in $\mu$-dish $35 \mathrm{~mm}$ with an ibidi polymer coverslip bottom (Gräfelfing, Germany). After incubation with MEM $\alpha$ supplemented with RANKL ( $300 \mathrm{ng} / \mathrm{mL}$ ) at $37^{\circ} \mathrm{C}$ in $5 \% \mathrm{CO}_{2}$ for $48 \mathrm{~h}$, cells were successively washed with serum-free MEM $\alpha$ twice, and incubated for 60 min with the same medium composed of $1 \mu \mathrm{M}$ Cal-590, a red-shifted $\mathrm{Ca}^{2+}$ indicator whose spectra of fluorescent emission is distinct from those of FITC, Alexa Flour 488, and GFP. Consequently, Cal-590 was ideally used to capture the distribution of $\mathrm{Ca}^{2+}$ signals in RAW-D cells expressing GFP with green fluorescence. The cells were then washed and analyzed using Olympus UPLSAPO 10X. The $\left(\mathrm{Ca}^{2+}{ }_{i}\right)$ fluorescent intensities were measured and analyzed by Image J software.

\subsection{Statistical Analysis}

Unless otherwise noted, each sample was assayed in triplicate. For in vitro analyses, each experiment was thrice repeated. Statistical analysis was performed using Student's $t$-test to compare two groups of independent samples ${ }^{*} p<0.05,{ }^{* *} p<0.01$, and ${ }^{* * *} p<0.001$, n.s., nonsignificant). The level of statistical significance was set at 0.05 for all tests.

Supplementary Materials: Supplementary materials can be found at http://www.mdpi.com/1422-0067/21/24/ 9352/s1.

Author Contributions: M.T.T. performed most of the experiments; Y.O. performed qPCR experiments, and gave technical assistance; Y.F. constructed retrovirus vectors and cloned RAW-D cells stably expressing GFP and GFP-Rab11b under supervision of K.O.; M.M., K.N., H.O., and M.T.T. performed $\left(\mathrm{Ca}^{2+}{ }_{\mathrm{i}}\right)$ fluorescent experiments; C.S., T.E., and P.W. gave technical assistance; T.K., E.S., and T.T. analyzed data and discussed the results; M.T.T. and K.O. designed the experiments, interpreted data, carried out the formal analysis, and wrote the manuscript. All authors have read and agreed to the published version of the manuscript.

Funding: This research was funded by JSPS KAKENHI Grant Number JP16K11863 (K.O.).

Conflicts of Interest: The authors declare no conflict of interest.

\section{Abbreviations}

BMMs

MNC

RANKL

M-CSF

MEM
Bone marrow-derived macrophages

Multinucleated cell

Nuclear factor $\kappa-B$ ligand

Macrophage colony-stimulating factor

Minimum essential medium 


$\begin{array}{ll}\text { NF- } к \text { B } & \text { Nuclear factor kappa B } \\ \text { MAPK } & \text { Mitogen-activated protein kinase } \\ \text { JNK } & \text { Jun N-terminal kinase } \\ \text { CTSK } & \text { Cathepsin K } \\ \text { LAMP1 } & \text { Lysosomal associated membrane protein 1 } \\ \text { PI3K/Akt } & \text { Phosphatidylinositol 3-kinase } \\ \text { ERK } & \text { Extracellular signal-regulated kinase } \\ \text { DAPI } & 4^{\prime} \text {,6-diamidino-2-phenylindole } \\ \text { SDS-PAGE } & \text { Sodium dodecyl sulphate-polyacrylamide gel electrophoresis }\end{array}$

\section{References}

1. Boyle, W.J.; Simonet, W.S.; Lacey, D.L. Osteoclast differentiation and activation. Nature 2003, 423, 337-342. [CrossRef]

2. Väänänen, H.K.; Zhao, H.; Mulari, M.; Halleen, J.M. The cell biology of osteoclast function. J. Cell Sci. 2000, 113 (Pt 3), 377-381. [PubMed]

3. Kim, J.H.; Kim, N. Signaling Pathways in Osteoclast Differentiation. Chonnam Med. J. 2016, 52, 12-17. [CrossRef] [PubMed]

4. Ballanti, P.; Minisola, S.; Pacitti, M.T.; Scarnecchia, L.; Rosso, R.; Mazzuoli, G.F.; Bonucci, E. Tartrate-resistant acid phosphate activity as osteoclastic marker: Sensitivity of cytochemical assessment and serum assay in comparison with standardized osteoclast histomorphometry. Osteoporos. Int. 1997, 7, 39-43. [CrossRef] [PubMed]

5. $\quad$ Everts, V.; Korper, W.; Hoeben, K.A.; Jansen, I.D.; Bromme, D.; Cleutjens, K.B.; Heeneman, S.; Peters, C.; Reinheckel, T.; Saftig, P.; et al. Osteoclastic Bone Degradation and the Role of Different Cysteine Proteinases and Matrix Metalloproteinases: Differences Between Calvaria and Long Bone. J. Bone Miner. Res. 2006, 21, 1399-1408. [CrossRef] [PubMed]

6. Feng, X.; Teitelbaum, S.L. Osteoclasts: New Insights. Bone Res. 2013, 1, 11-26. [CrossRef] [PubMed]

7. Li, G.; Marlin, M.C. Rab Family of GTPases. Methods Mol. Biol. 2015, 1298, 1-15. [CrossRef] [PubMed]

8. Stenmark, H. Rab GTPases as coordinators of vesicle traffic. Nat. Rev. Mol. Cell Biol. 2009, 10, 513-525. [CrossRef] [PubMed]

9. Cherfils, J.; Zeghouf, M. Regulation of Small GTPases by GEFs, GAPs, and GDIs. Physiol. Rev. 2013, 93, 269-309. [CrossRef]

10. Kotting, C.; Gerwert, K. The dynamics of the catalytic site in small GTPases, variations on a common motif. FEBS Lett. 2013, 587, 2025-2027. [CrossRef]

11. Wandinger-Ness, A.; Zerial, M. Rab Proteins and the Compartmentalization of the Endosomal System. Cold Spring Harb. Perspect. Biol. 2014, 6, a022616. [CrossRef] [PubMed]

12. Wu, X.; Bradley, M.J.; Cai, Y.; Kummel, D.; De La Cruz, E.M.; Barr, F.A.; Reinisch, K.M. Insights regarding guanine nucleotide exchange from the structure of a DENN-domain protein complexed with its Rab GTPase substrate. Proc. Natl. Acad. Sci. USA 2011, 108, 18672-18677. [CrossRef] [PubMed]

13. Bhartur, S.G.; Calhoun, B.C.; Woodrum, J.; Kurkjian, J.; Iyer, S.; Lai, F.; Goldenring, J.R. Genomic Structure of Murine Rab11 Family Members. Biochem. Biophys. Res. Commun. 2000, 269, 611-617. [CrossRef] [PubMed]

14. Lapierre, L.A.; Dorn, M.C.; Zimmerman, C.F.; Navarre, J.; Burnette, J.O.; Goldenring, J.R. Rab11b resides in a vesicular compartment distinct from Rab11a in parietal cells and other epithelial cells. Exp. Cell Res. 2003, 290, 322-331. [CrossRef] [PubMed]

15. Krishnan, M.; Lapierre, L.A.; Knowles, B.C.; Goldenring, J.R. Rab25 regulates integrin expression in polarized colonic epithelial cells. Mol. Biol. Cell 2013, 24, 818-831. [CrossRef]

16. Yamaguchi, Y.; Sakai, E.; Okamoto, K.; Kajiya, H.; Okabe, K.; Naito, M.; Kadowaki, T.; Tsukuba, T. Rab44, a novel large Rab GTPase, negatively regulates osteoclast differentiation by modulating intracellular calcium levels followed by NFATc1 activation. Cell. Mol. Life Sci. 2018, 75, 33-48. [CrossRef]

17. Shimada-Sugawara, M.; Sakai, E.; Okamoto, K.; Fukuda, M.; Izumi, T.; Yoshida, N.; Tsukuba, T. Rab27A Regulates Transport of Cell Surface Receptors Modulating Multinucleation and Lysosome-Related Organelles in Osteoclasts. Sci. Rep. 2015, 5, 9620. [CrossRef] 
18. Okusha, Y.; Tran, M.T.; Itagaki, M.; Sogawa, C.; Eguchi, T.; Okui, T.; Kadowaki, T.; Sakai, E.; Tsukuba, T.; Okamoto, K. Rab11A Functions as a Negative Regulator of Osteoclastogenesis through Dictating Lysosome-Induced Proteolysis of c-fms and RANK Surface Receptors. Cells 2020, 9, 2384. [CrossRef]

19. Huang, H.; Chang, E.J.; Ryu, J.; Lee, Z.H.; Lee, Y.; Kim, H.H. Induction of c-Fos and NFATc1 during RANKL-stimulated osteoclast differentiation is mediated by the p38 signaling pathway. Biochem. Biophys. Res. Commun. 2006, 351, 99-105. [CrossRef]

20. Kim, K.; Kim, J.H.; Lee, J.; Jin, H.M.; Lee, S.H.; Fisher, D.E.; Kook, H.; Kim, K.K.; Choi, Y.; Kim, N. Nuclear Factor of Activated T Cells c1 Induces Osteoclast-associated Receptor Gene Expression during Tumor Necrosis Factor-related Activation-induced Cytokine-mediated Osteoclastogenesis. J. Biol. Chem. 2005, 280, 35209-35216. [CrossRef]

21. Matsumoto, M.; Kogawa, M.; Wada, S.; Takayanagi, H.; Tsujimoto, M.; Katayama, S.; Hisatake, K.; Nogi, Y. Essential Role of p38 Mitogen-activated Protein Kinase in Cathepsin K Gene Expression during Osteoclastogenesis through Association of NFATc1 and PU.1. J. Biol. Chem. 2004, 279, 45969-45979. [CrossRef] [PubMed]

22. Takayanagi, H.; Kim, S.; Koga, T.; Nishina, H.; Isshiki, M.; Yoshida, H.; Saiura, A.; Isobe, M.; Yokochi, T.; Inoue, J.; et al. Induction and Activation of the Transcription Factor NFATc1 (NFAT2) Integrate RANKL Signaling in Terminal Differentiation of Osteoclasts. Dev. Cell 2002, 3, 889-901. [CrossRef]

23. Hogan, P.G.; Chen, L.; Nardone, J.; Rao, A. Transcriptional regulation by calcium, calcineurin, and NFAT. Genes Dev. 2003, 17, 2205-2232. [CrossRef]

24. Chen, S.; Pan, M. NFAT Signaling and Bone Homeostasis. J. Hematol. Thromboembolic Dis. 2013, 1. [CrossRef]

25. Morgan, A.J.; Jacob, R. Ionomycin enhances Ca2+ influx by stimulating store-regulated cation entry and not by a direct action at the plasma membrane. Biochem. J. 1994, 300 (Pt 3), 665-672. [CrossRef] [PubMed]

26. Schlierf, B.; Fey, G.H.; Hauber, J.; Hocke, G.M.; Rosorius, O. Rab11b Is Essential for Recycling of Transferrin to the Plasma Membrane. Exp. Cell Res. 2000, 259, 257-265. [CrossRef] [PubMed]

27. Al-Bari, A.; Shinohara, M.; Nagai, Y.; Takayanagi, H. Inhibitory effect of chloroquine on bone resorption reveals the key role of lysosomes in osteoclast differentiation and function. Inflamm. Regen. 2012, 32, 222-231. [CrossRef]

28. Erkhembaatar, M.; Gu, D.R.; Lee, S.H.; Yang, Y.-M.; Park, S.; Muallem, S.; Shin, D.M.; Kim, M.S. Lysosomal $\mathrm{Ca}(2+)$ Signaling is Essential for Osteoclastogenesis and Bone Remodeling. J. Bone Miner. Res. 2017, 32, 385-396. [CrossRef]

29. Lacombe, J.; Karsenty, G.; Ferron, M. Regulation of lysosome biogenesis and functions in osteoclasts. Cell Cycle 2013, 12, 2744-2752. [CrossRef]

30. Dikic, I. Proteasomal and Autophagic Degradation Systems. Annu. Rev. Biochem. 2017, 86, 193-224. [CrossRef]

31. Lecker, S.H.; Goldberg, A.L.; Mitch, W.E. Protein Degradation by the Ubiquitin-Proteasome Pathway in Normal and Disease States. J. Am. Soc. Nephrol. 2006, 17, 1807-1819. [CrossRef] [PubMed]

32. Luzio, J.P.; Pryor, P.R.; Bright, N.A. Lysosomes: Fusion and function. Nat. Rev. Mol. Cell Biol. 2007, 8, 622-632. [CrossRef] [PubMed]

33. Naslavsky, N.; Caplan, S. The enigmatic endosome-sorting the ins and outs of endocytic trafficking. J. Cell Sci. 2018, 131, jcs216499. [CrossRef] [PubMed]

34. Van Der Goot, F.G.; Gruenberg, J. Intra-endosomal membrane traffic. Trends Cell Biol. 2006, 16, 514-521. [CrossRef]

35. Song, I.; Kim, J.H.; Kim, K.; Jin, H.M.; Youn, B.U.; Kim, N. Regulatory mechanism of NFATc1 in RANKL-induced osteoclast activation. FEBS Lett. 2009, 583, 2435-2440. [CrossRef]

36. Sundaram, K.; Nishimura, R.; Senn, J.; Youssef, R.F.; London, S.D.; Reddy, S.V. RANK ligand signaling modulates the matrix metalloproteinase-9 gene expression during osteoclast differentiation. Exp. Cell Res. 2007, 313, 168-178. [CrossRef]

37. Zulkefli, K.L.; Houghton, F.J.; Gosavi, P.; Gleeson, P.A. A role for Rab11 in the homeostasis of the endosome-lysosomal pathway. Exp. Cell Res. 2019, 380, 55-68. [CrossRef]

38. Copp, D.H.; Cheney, B. Calcitonin-A Hormone from the Parathyroid which Lowers the Calcium-level of the Blood. Nature 1962, 193, 381-382. [CrossRef]

39. Foster, G.V.; Baghdiantz, A.; Kumar, M.A.; Slack, E.; Soliman, H.A.; MacIntyre, I. Thyroid origin of Calcitonin. Nature 1964, 202, 1303-1305. [CrossRef] 
40. Alam, A.S.; Bax, C.M.; Shankar, V.S.; Bax, B.E.; Bevis, P.J.; Huang, C.L.; Moonga, B.S.; Pazianas, M.; Zaidi, M.; Baumrucker, C.R.; et al. Further studies on the mode of action of calcitonin on isolated rat osteoclasts: Pharmacological evidence for a second site mediating intracellular Ca2+ mobilization and cell retraction. J. Endocrinol. 1993, 136, 7-15. [CrossRef]

41. Chambers, T.J.; Moore, A. The Sensitivity of Isolated Osteoclasts to Morphological Transformation by Calcitonin. J. Clin. Endocrinol. Metab. 1983, 57, 819-824. [CrossRef] [PubMed]

42. Suzuki, H.; Nakamura, I.; Takahashi, N.; Ikuhara, T.; Matsuzaki, K.; Isogai, Y.; Hori, M.; Suda, T. Calcitonin-induced changes in the cytoskeleton are mediated by a signal pathway associated with protein kinase A in osteoclasts. Endocrinology 1996, 137, 4685-4690. [CrossRef]

43. Granholm, S.; Lundberg, P.; Lerner, U.H. Expression of the calcitonin receptor, calcitonin receptor-like receptor, and receptor activity modifying proteins during osteoclast differentiation. J. Cell. Biochem. 2008, 104, 920-933. [CrossRef]

44. Lerner, U.H. Deletions of genes encoding calcitonin/alpha-CGRP, amylin and calcitonin receptor have given new and unexpected insights into the function of calcitonin receptors and calcitonin receptor-like receptors in bone. J. Musculoskelet. Neuronal Interact. 2006, 6, 87-95. [PubMed]

45. Arai, F.; Miyamoto, T.; Ohneda, O.; Inada, T.; Sudo, T.; Brasel, K.; Miyata, T.; Anderson, D.M.; Suda, T. Commitment and Differentiation of Osteoclast Precursor Cells by the Sequential Expression of C-Fms and Receptor Activator of Nuclear Factor kb (Rank) Receptors. J. Exp. Med. 1999, 190, 1741-1754. [CrossRef] [PubMed]

46. Choi, Y.; Faccio, R.; Teitelbaum, S.L.; Takayanagi, H. Osteoclast Biology: Regulation of Formation and Function. In Osteoimmunology; Academic Press: Cambridge, MA, USA, 2016; pp. 41-70. [CrossRef]

47. Feng, X. RANKing Intracellular Signaling in Osteoclasts. IUBMB Life 2005, 57, 389-395. [CrossRef]

48. Miyamoto, T.; Suda, T. Differentiation and function of osteoclasts. Keio J. Med. 2003, 52, 1-7. [CrossRef]

49. Hienz, S.A.; Paliwal, S.; Ivanovski, S. Mechanisms of Bone Resorption in Periodontitis. J. Immunol. Res. 2015, 2015, 615486. [CrossRef]

50. Karmakar, S.; Kay, J.; Gravallese, E.M. Bone Damage in Rheumatoid Arthritis: Mechanistic Insights and Approaches to Prevention. Rheum. Dis. Clin. N. Am. 2010, 36, 385-404. [CrossRef]

51. Rachner, T.D.; Khosla, S.; Hofbauer, L.C. Osteoporosis: Now and the future. Lancet 2011, 377, $1276-1287$. [CrossRef]

52. Sabharwal, R.; Gupta, S.; Sepolia, S.; Panigrahi, R.; Mohanty, S.; Subudhi, S.K.; Kumar, M. An Insight in to Paget's Disease of Bone. Niger. J. Surg. 2014, 20, 9-15. [CrossRef] [PubMed]

53. Zupan, J.; Komadina, R.; Marc, J. The relationship between osteoclastogenic and anti-osteoclastogenic pro-inflammatory cytokines differs in human osteoporotic and osteoarthritic bone tissues. J. Biomed. Sci. 2012, 19, 28. [CrossRef]

54. Sakai, E.; Shimada-Sugawara, M.; Nishishita, K.; Fukuma, Y.; Naito, M.; Okamoto, K.; Nakayama, K.; Tsukuba, T. Suppression of RANKL-dependent heme oxygenase-1 is required for high mobility group box 1 release and osteoclastogenesis. J. Cell. Biochem. 2012, 113, 486-498. [CrossRef] [PubMed]

55. Nakanishi, H.; Tominaga, K.; Amano, T.; Hirotsu, I.; Inoue, T.; Yamamoto, K. Age-Related Changes in Activities and Localizations of Cathepsins D, E, B, and L in the Rat Brain Tissues. Exp. Neurol. 1994, 126, 119-128. [CrossRef] [PubMed]

56. Kukita, T.; Wada, N.; Kukita, A.; Kakimoto, T.; Sandra, F.; Toh, K.; Nagata, K.; Iijima, T.; Horiuchi, M.; Matsusaki, H.; et al. RANKL-induced DC-STAMP Is Essential for Osteoclastogenesis. J. Exp. Med. 2004, 200, 941-946. [CrossRef] [PubMed]

57. Watanabe, T.; Kukita, T.; Kukita, A.; Wada, N.; Toh, K.; Nagata, K.; Nomiyama, H.; Iijima, T. Direct stimulation of osteoclastogenesis by MIP-1alpha: Evidence obtained from studies using RAW264 cell clone highly responsive to RANKL. J. Endocrinol. 2004, 180, 193-201. [CrossRef] [PubMed]

Publisher's Note: MDPI stays neutral with regard to jurisdictional claims in published maps and institutional affiliations. 